\title{
Properties of excited charmed-bottom mesons
}

\author{
Ishrat Asghar®, ${ }^{*}$ Faisal Akram $\odot,^{\dagger}$ Bilal Masud, ${ }^{\dagger}$ and M. Atif Sultan ${ }^{\S}$ \\ Center For High Energy Physics, Punjab University, Lahore 54590, Pakistan
}

(Received 8 August 2019; published 8 November 2019)

\begin{abstract}
We calculate the spectrum of $B_{c}$ mesons using a nonrelativistic quark potential model. Using the calculated wave functions, we compute the radiative widths of $B_{c}$ excited states. The strong decay widths are calculated in a modified ${ }^{3} P_{0}$ model, assuming harmonic oscillator wave functions. The hadronic transition rates of $B_{c}$ mesons are calculated using the Kuang-Yan approach. These results are used to determine branching ratios of possible decay channels of several $B_{c}$ excited states. Calculated branching ratios are then combined with production cross section of $B_{c}$ states at the LHC to suggest strategies to find missing excited states of $B_{c}$ mesons.
\end{abstract}

DOI: 10.1103/PhysRevD.100.096002

\section{INTRODUCTION}

The $B_{c}^{+}$meson is the lowest-mass bound state of a charm quark and a bottom antiquark. This pseudoscalar mesonic ground state has no electromagnetic or strong decays as it cannot annihilate into gluons or photons. It was first observed by the CDF Collaboration at Fermilab through a semileptonic decay mode $B_{c}^{ \pm} \rightarrow J / \psi l^{ \pm} \nu$ in $p \bar{p}$ collisions. The measured mass and life time of $B_{c}$ by CDF were $6.40 \pm 0.39 \pm 0.13 \mathrm{GeV}$ and $0.46_{-0.16}^{+0.18} \pm 0.03 \mathrm{ps}$ respectively [1]. It has also been observed by LHCb [2] and D0 [3] experiments through its different decay channels. In 2014 an excited state of $B_{c}$ meson was observed by ATLAS experiment at LHC through the decay channel $B_{c}^{ \pm} \rightarrow J / \psi \pi^{ \pm}$in $p p$ collisions. The measured mass of the excited state was found to be $6842 \pm 4 \pm 5 \mathrm{MeV}$ [4], which is considered the second $\mathrm{S}$-wave state of $B_{c}$. However, this excited state has not been confirmed by other experiments yet. Excited $B_{c}$ states below $B D$ threshold $(\approx 7144 \mathrm{MeV})$ can only decay through radiative and hadronic transitions to $B_{c}$ ground state, which decay through weak interaction. There are at least two $S$-wave, two $P$-wave, and one $D$-wave $B_{c}$ multiplets lying below the threshold. Each of these states cascades into $B_{c}$ ground state through emission of photons and/or pions only. This results into unique experimental signatures through which

\footnotetext{
*ishrat.2000@gmail.com

faisal.chep@pu.edu.pk

tbilalmasud.chep@pu.edu.pk

§atifsultan.chep@pu.edu.pk
}

Published by the American Physical Society under the terms of the Creative Commons Attribution 4.0 International license. Further distribution of this work must maintain attribution to the author(s) and the published article's title, journal citation, and DOI. Funded by SCOAP ${ }^{3}$. we can identify them. This is particularly important when a large sample of $B_{c}$ states is expected to be produced at the LHC. To predict event rates of various decay chains of excited $B_{c}$ states lying below the $B D$ threshold at the LHC, we require a knowledge of branching ratios of their electromagnetic and hadronic transitions along with their production cross sections.

There have been many calculations of the $B_{c}$ spectrum using nonrelativistic and relativistic quark models [5-10]. The electromagnetic transitions of $B_{c}$ are predicted in Refs. [6,8-11] and hadronic transitions are calculated in $[8,12]$. In Ref. [5], the open-flavor strong decay widths of $B_{c}$ mesons are predicted in the ${ }^{3} P_{0}$ model. Reference [5] studied only strong decays to pairs of $\mathrm{S}$-wave mesons for many open flavor states, using the same value of the harmonic oscillator parameter $\beta$ for all the flavor states. The present work provides a comprehensive theoretical study of $B_{c}$ mesons properties: Here we calculate the spectrum, radiative transitions ( $E 1$ and $M 1)$, hadronic transitions, and strong decays of $B_{c}$ mesons following a consistent approach. A nonrelativistic potential quark model is used to explain the mass spectrum of $B_{c}$ mesons. The wave functions computed through this model are then used to find the decay widths of their $E 1$ and $M 1$ transitions. The hadronic transitions of $B_{c}$ mesons are estimated by using the Kuang-Yan approach. We use the ${ }^{3} P_{0}$ model to study the open-flavor strong decays of $B_{c}$ states. In comparison to Ref. [5], we have calculated strong decay widths of all angularly excited $B_{c}$ states while using different values of $\beta$ for different flavor states. We combine radiative, hadronic, and strong widths to predict the branching ratios of all possible decay channels of several $B_{c}$ excited states. The branching ratios of radiative and hadronic transitions of $B_{c}$ excited states lying below the $B D$ threshold are combined with their predicted production cross sections at the LHC energy to provide estimates of event rates of their possible 
decay chains through which these states can be identified in the experimental data.

The organization of the paper is as follows. First, we describe the potential model used to calculate the mass spectrum of charm-bottom mesons. In Sec. III, we review the ${ }^{3} P_{0}$ decay model and evaluate the strong decay amplitudes. $E 1$ and $M 1$ radiative transitions are calculated in Sec. IV. This is followed by the estimates in Sec. V of hadronic transitions based on the Kuang-Yan approach. We discuss the best strategies for searching the excited $B_{c}$ states lying below the $B D$ threshold in Sec. VI, while our concluding remarks are given in Sec. VII.

\section{MASS SPECTRUM}

In this section we give the mass predictions of the nonrelativistic quark model for charm-bottom mesons. We use the standard "Coulomb + linear" potential, and spin-dependent corrections generated from vector gluon exchange and an effective scalar confinement interaction. The potential used in this paper is given by [13]

$$
\begin{aligned}
V_{q \bar{q}}(r)= & -\frac{4 \alpha_{s}}{3 r}+b r+\frac{32 \pi \alpha_{s}}{9 m_{q} m_{\bar{q}}}\left(\frac{\sigma}{\sqrt{\pi}}\right)^{3} e^{-\sigma^{2} r^{2} \mathbf{S}_{q} \cdot \mathbf{S}_{\bar{q}}} \\
& +\frac{4 \alpha_{s}}{m_{q} m_{\bar{q}} r^{3}} T+\left(\frac{\mathbf{S}_{q}}{4 m_{q}^{2}}+\frac{\mathbf{S}_{\bar{q}}}{4 m_{\bar{q}}^{2}}\right) \cdot \mathbf{L}\left(\frac{4 \alpha_{s}}{3 r^{3}}-\frac{b}{r}\right) \\
& +\frac{\mathbf{S}_{q}+\mathbf{S}_{\bar{q}}}{2 m_{q} m_{\bar{q}}} \cdot \mathbf{L} \frac{4 \alpha_{s}}{3 r^{3}} .
\end{aligned}
$$

Here $\alpha_{s}$ is the strong coupling constant, $b$ is the string tension, and $T$ is the tensor operator

$$
T=\mathbf{S}_{q} \cdot \hat{r} \mathbf{S}_{\bar{q}} \cdot \hat{r}-\frac{1}{3} \mathbf{S}_{q} \cdot \mathbf{S}_{\bar{q}},
$$

with diagonal matrix elements given by

$$
T= \begin{cases}-\frac{L}{6(2 L+3)} & J=L+1 \\ \frac{1}{6} & J=L \\ -\frac{(L+1)}{6(2 L-1)} & J=L-1 .\end{cases}
$$

The strong coupling constant $\alpha_{s}$ used in this potential for $B_{c}$ mesons is taken to be 0.4 . This value was obtained by our fit to the masses of two experimentally known states of $B_{c}$ mesons (these are listed in the fourth column of Table I). The parameters $\sigma=0.84 \mathrm{GeV}, b=0.0945 \mathrm{GeV}^{2}$, $m_{u / d}=0.325 \mathrm{GeV}$, and $m_{s}=0.422 \mathrm{GeV}$ were obtained from fits to light mesons [14], and $m_{c}=1.4794 \mathrm{GeV}$ is from the charmonium sector. Finally $m_{b}=4.825 \mathrm{GeV}$ is taken from Ref. [15].

The meson states with quark and antiquark of unequal mass are not charge conjugation eigenstates. Therefore, states with $J=L$ and $S=0,1$, i.e., $\left|n^{1} L_{J}\right\rangle$ and $\left|n^{3} L_{J}\right\rangle$ can mix via spin-orbit interaction. For example ${ }^{1} P_{1}$ and ${ }^{3} P_{1}$ states can mix through the following linear combination:

$$
\begin{aligned}
& \left|B\left(1 P_{1}\right)\right\rangle=+\cos \left(\phi_{1 P}\right)\left|1^{1} P_{1}\right\rangle+\sin \left(\phi_{1 P}\right)\left|1^{3} P_{1}\right\rangle, \\
& \left|B\left(1 P_{1}^{\prime}\right)\right\rangle=-\sin \left(\phi_{1 P}\right)\left|1^{1} P_{1}\right\rangle+\cos \left(\phi_{1 P}\right)\left|1^{3} P_{1}\right\rangle,
\end{aligned}
$$

where $\phi_{1 P}$ is the mixing angle. In the heavy quark limit $m_{Q} \rightarrow \infty$ the mixing angles become [17]

$$
\phi_{m_{Q} \rightarrow \infty}=\arctan \left(\sqrt{\frac{L}{L+1}}\right) .
$$

This implies $\phi_{n P}=35.3^{\circ}, \phi_{n D}=39.2^{\circ}$, and $\phi_{n F}=40.89^{\circ}$. The mixing angles in the heavy quark limit for $1 D$ and $1 F$ states are close to those produced by Ref. [8] whereas for the $1 P$ state it is slightly different. The spectrum of $B_{c}$ states obtained by solving the radial Schrödinger equation through the shooting method [18] is reported in Tables I and II.

\section{OPEN FLAVOR STRONG DECAYS}

In the ${ }^{3} P_{0}$ model, the open-flavor strong decay of a meson takes place through production of a light $q \bar{q}$ pair $(q=u, d, s)$ with vacuum quantum numbers $\left(J^{P C}=0^{++}\right)$. The interaction Hamiltonian for the ${ }^{3} P_{0}$ model is obtained from the nonrelativistic limit of

$$
H_{I}=2 m_{q} \gamma \int d^{3} \mathbf{x} \bar{\psi}_{q}(\mathbf{x}) \psi_{q}(\mathbf{x})
$$

where $\gamma$ is a dimensionless pair production strength. The pair-production strength parameter $\gamma$ is fitted to strong decay data. In the original ${ }^{3} P_{0}$ model introduced by Micu [19], a new $q \bar{q}$ pair is produced by a constant pair production amplitude $\gamma$. Some variants of the ${ }^{3} P_{0}$ model include an effective pair production strength $\left(\gamma^{\text {eff }}\right)$ that suppresses heavy $q \bar{q}$ pair production [20,21].

The ${ }^{3} P_{0}$ model has been successfully applied to strong decays of light mesons [22], strange mesons [23,24], charmonium states [13], bottomonium states [25,26], open-charm [27,28], and open-bottom sectors [5,14,29, 30]. In this study, we have computed strong decay widths of kinematically allowed open-flavor decay modes of all the $B_{c}$ states mentioned in Tables I and II using the ${ }^{3} P_{0}$ model. The interaction Hamiltonian for the ${ }^{3} P_{0}$ model can be written in terms of the creation operators as

$H_{I}=2 m_{q} \gamma \int d^{3} k[\bar{u}(\mathbf{k}, s) v(-\mathbf{k}, \bar{s})] b^{\dagger}(\mathbf{k}, s) d^{\dagger}(-\mathbf{k}, \bar{s})$,

where $b^{\dagger}$ and $d^{\dagger}$ are the creation operators for quark and antiquark respectively. The pair production strength factor 
TABLE I. Masses of ground and excited states of $B_{c}$ mesons. The mixed states $P_{1}-P_{1}^{\prime}, D_{2}-D_{2}^{\prime}$, and $F_{3}-F_{3}^{\prime}$ and their mixing angles $\phi_{n P}, \phi_{n D}$, and $\phi_{n F}$ are defined according to Eq. (5). The SHO $\beta$ values are listed in the last column which are obtained by fitting SHO wave functions to the quark model wave functions.

\begin{tabular}{|c|c|c|c|c|}
\hline $\mathrm{nL}$ & Meson & Our calculated mass $(\mathrm{GeV})$ & Expt. mass [16] (MeV) & $\beta(\mathrm{GeV})$ \\
\hline $1 \mathrm{~S}$ & $\begin{array}{l}B_{c}\left(1^{1} S_{0}\right) \\
B_{c}\left(1^{3} S_{1}\right)\end{array}$ & $\begin{array}{l}6.318 \\
6.336\end{array}$ & $\begin{array}{c}6274.9 \pm 0.8 \\
\ldots\end{array}$ & $\begin{array}{l}0.653 \\
0.634\end{array}$ \\
\hline $2 \mathrm{~S}$ & $\begin{array}{l}B_{c}\left(2^{1} S_{0}\right) \\
B_{c}\left(2^{3} S_{1}\right)\end{array}$ & $\begin{array}{l}6.741 \\
6.747\end{array}$ & $\begin{array}{c}6842 \pm 4 \\
\ldots\end{array}$ & $\begin{array}{l}0.515 \\
0.508\end{array}$ \\
\hline $3 \mathrm{~S}$ & $\begin{array}{l}B_{c}\left(3{ }^{1} S_{0}\right) \\
B_{c}\left(3^{3} S_{1}\right)\end{array}$ & $\begin{array}{l}7.014 \\
7.018\end{array}$ & $\begin{array}{l}\cdots \\
\cdots\end{array}$ & $\begin{array}{l}0.442 \\
0.439\end{array}$ \\
\hline $4 \mathrm{~S}$ & $\begin{array}{l}B_{c}\left(4^{1} S_{0}\right) \\
B_{c}\left(4^{3} S_{1}\right)\end{array}$ & $\begin{array}{l}7.239 \\
7.242\end{array}$ & $\begin{array}{l}\cdots \\
\cdots\end{array}$ & $\begin{array}{l}0.402 \\
0.401\end{array}$ \\
\hline $1 \mathrm{P}$ & $\begin{array}{c}B_{c}\left(1^{3} P_{0}\right) \\
B_{c}\left(1^{3} P_{2}\right) \\
B_{c}\left(1 P_{1}\right) \\
B_{c}\left(1 P_{1}^{\prime}\right) \\
\quad \phi_{1 P}\end{array}$ & $\begin{array}{l}6.631 \\
6.665 \\
6.650 \\
6.656 \\
35.3^{\circ}\end{array}$ & $\begin{array}{l}\cdots \\
\cdots \\
\cdots \\
\cdots\end{array}$ & $\begin{array}{c}0.468 \\
0.468 \\
0.471,0.468 \\
0.471,0.468\end{array}$ \\
\hline $2 \mathrm{P}$ & $\begin{array}{c}B_{c}\left(2^{3} P_{0}\right) \\
B_{c}\left(2^{3} P_{2}\right) \\
B_{c}\left(2 P_{1}\right) \\
B_{c}\left(2 P_{1}^{\prime}\right) \\
\phi_{2 P}\end{array}$ & $\begin{array}{l}6.915 \\
6.946 \\
6.930 \\
6.939 \\
35.3^{\circ}\end{array}$ & $\begin{array}{l}\cdots \\
\cdots \\
\cdots \\
\cdots \\
\cdots\end{array}$ & $\begin{array}{c}0.428 \\
0.428 \\
0.430,0.428 \\
0.430,0.428\end{array}$ \\
\hline $3 \mathrm{P}$ & $\begin{array}{c}B_{c}\left(3^{3} P_{0}\right) \\
B_{c}\left(3^{3} P_{2}\right) \\
B_{c}\left(3 P_{1}\right) \\
B_{c}\left(3 P_{1}^{\prime}\right) \\
\phi_{3 P}\end{array}$ & $\begin{array}{l}7.147 \\
7.176 \\
7.162 \\
7.168 \\
35.3^{\circ}\end{array}$ & $\begin{array}{l}\cdots \\
\cdots \\
\cdots \\
\cdots \\
\cdots\end{array}$ & $\begin{array}{c}0.395 \\
0.395 \\
0.397,0.395 \\
0.397,0.395\end{array}$ \\
\hline $4 \mathrm{P}$ & $\begin{array}{c}B_{c}\left(4^{3} P_{0}\right) \\
B_{c}\left(4^{3} P_{2}\right) \\
B_{c}\left(4 P_{1}\right) \\
B_{c}\left(4 P_{1}^{\prime}\right) \\
\quad \phi_{4 P}\end{array}$ & $\begin{array}{l}7.350 \\
7.379 \\
7.364 \\
7.373 \\
35.3^{\circ}\end{array}$ & $\begin{array}{l}\cdots \\
\cdots \\
\cdots \\
\cdots \\
\cdots\end{array}$ & $\begin{array}{c}0.373 \\
0.373 \\
0.374,0.373 \\
0.374,0.373\end{array}$ \\
\hline $1 \mathrm{D}$ & $\begin{array}{c}B_{c}\left(1^{3} D_{1}\right) \\
B_{c}\left(1^{3} D_{3}\right) \\
B_{c}\left(1 D_{2}\right) \\
B_{c}\left(1 D_{2}^{\prime}\right) \\
\quad \phi_{1 D}\end{array}$ & $\begin{array}{l}6.841 \\
6.847 \\
6.845 \\
6.845 \\
39.2^{\circ}\end{array}$ & $\begin{array}{l}\cdots \\
\cdots \\
\cdots \\
\cdots \\
\cdots\end{array}$ & $\begin{array}{c}0.417 \\
0.417 \\
0.417,0.417 \\
0.417,0.417\end{array}$ \\
\hline $2 \mathrm{D}$ & $\begin{array}{c}B_{c}\left(2^{3} D_{1}\right) \\
B_{c}\left(2^{3} D_{3}\right) \\
B_{c}\left(2 D_{2}\right) \\
B_{c}\left(2 D_{2}^{\prime}\right) \\
\phi_{2 D}\end{array}$ & $\begin{array}{l}7.080 \\
7.087 \\
7.084 \\
7.084 \\
39.2^{\circ}\end{array}$ & $\begin{array}{l}\cdots \\
\cdots \\
\cdots \\
\cdots \\
\cdots\end{array}$ & $\begin{array}{c}0.395 \\
0.395 \\
0.395,0.395 \\
0.395,0.395\end{array}$ \\
\hline $3 \mathrm{D}$ & $\begin{array}{c}B_{c}\left(3^{3} D_{1}\right) \\
B_{c}\left(3^{3} D_{3}\right) \\
B_{c}\left(3 D_{2}\right) \\
B_{c}\left(3 D_{2}^{\prime}\right) \\
\phi_{3 D}\end{array}$ & $\begin{array}{l}7.289 \\
7.296 \\
7.293 \\
7.293 \\
39.2^{\circ}\end{array}$ & $\begin{array}{l}\cdots \\
\cdots \\
\cdots \\
\cdots \\
\cdots\end{array}$ & $\begin{array}{c}0.374 \\
0.374 \\
0.374,0.374 \\
0.374,0.374\end{array}$ \\
\hline $4 \mathrm{D}$ & $\begin{array}{c}B_{c}\left(4^{3} D_{1}\right) \\
B_{c}\left(4^{3} D_{3}\right) \\
B_{c}\left(4 D_{2}\right) \\
B_{c}\left(4 D_{2}^{\prime}\right) \\
\phi_{4 D}\end{array}$ & $\begin{array}{l}7.478 \\
7.489 \\
7.482 \\
7.483 \\
39.2^{\circ}\end{array}$ & $\begin{array}{l}\cdots \\
\cdots \\
\cdots \\
\cdots \\
\cdots\end{array}$ & $\begin{array}{c}0.357 \\
0.357 \\
0.358,0.357 \\
0.358,0.357\end{array}$ \\
\hline
\end{tabular}


TABLE II. Masses of ground and excited states of $B_{c}$ mesons (continued).

\begin{tabular}{ccccc}
\hline \hline $\mathrm{nL}$ & Meson & Our calculated mass $(\mathrm{GeV})$ & Expt. mass $[16](\mathrm{MeV})$ & $\beta(\mathrm{GeV})$ \\
\hline $1 \mathrm{~F}$ & $B_{c}\left(1^{3} F_{2}\right)$ & 6.9972 & $\ldots$ & 0.390 \\
& $B_{c}\left(1^{3} F_{4}\right)$ & 6.9967 & $\ldots$ & 0.390 \\
& $B_{c}\left(1 F_{3}\right)$ & 6.994 & $\ldots$ & $0.390,0.390$ \\
& $B_{c}\left(1 F_{3}^{\prime}\right)$ & 7.001 & $\ldots$ & $0.390,0.390$ \\
& $\phi_{1 F}$ & $40.89^{\circ}$ & $\ldots$ & \\
& & 7.2121 & $\ldots$ & 0.375 \\
& $B_{c}\left(2^{3} F_{2}\right)$ & 7.2126 & $\ldots$ & 0.375 \\
& $B_{c}\left(2^{3} F_{4}\right)$ & 7.211 & $\ldots$ & $0.375,0.375$ \\
& $B_{c}\left(2 F_{3}\right)$ & $\ldots .214$ & $\ldots$ & \\
$B_{c}\left(2 F_{3}^{\prime}\right)$ & $40.89^{\circ}$ & $\ldots$ & \\
& $\phi_{2 F}$ & &
\end{tabular}

$\gamma=0.35$ is obtained from a fit of known strong decay widths of the $c \bar{c}$ states above open-charm threshold [13]. In this work, we use a modified version of pair production strength that replaces $\gamma$ with

$$
\gamma^{\mathrm{eff}}=\frac{m_{u / d}}{m} \gamma
$$

where $m$ is the mass of the produced quark [20,21]. This mechanism suppresses those diagrams in which a heavy $q \bar{q}$ pair is created.

With the ${ }^{3} P_{0}$ model, we use simple harmonic oscillator (SHO) wave functions and the $\mathrm{SHO}$ scale $\beta$ is taken as the parameter of the model. In Refs. [27-30], the $\beta$ parameter was obtained by equating the rootmean-squared (rms) radius of a harmonic oscillator wave function to the rms radius of the quark model wave function. In this work, we fit $\beta$ of SHO wave functions to the wave functions obtained by numerically

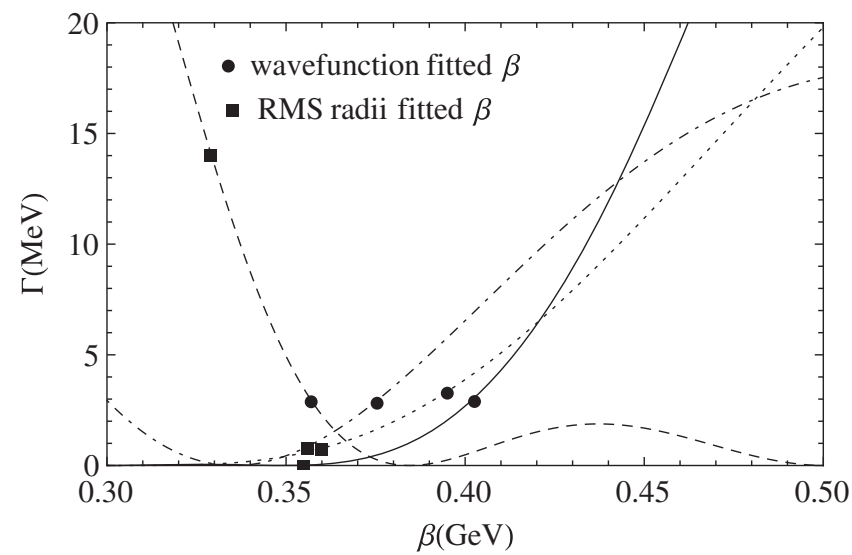

FIG. 1. Plots of partial widths of some of the strong decays of $B_{c}$ mesons in the ${ }^{3} P_{0}$ model as a function of $\beta$ for the initial meson. Solid curve: $B_{c}\left(4^{1} S_{0}\right) \rightarrow D B^{*}$; dotted curve: $B_{c}\left(3^{3} P_{0}\right) \rightarrow D B$; dashed curve: $B_{c}\left(4^{3} D_{1}\right) \rightarrow D B$; dot-dashed curve: $B_{c}\left(2^{3} F_{2}\right) \rightarrow D B$. Disk and rectangular marks on each curve correspond to the $\beta$ values obtained by the fit to numerically calculated wave functions and to rms radii respectively. solving the Schrödinger equation for the potential given in Eq. (1). The resulting $\beta$ values, that are more accurate, are listed in Tables I-III for the initial $B_{c}$ mesons, and the final $D, D_{s}, B$, and $B_{s}$ mesons appearing in strong decays of $B_{c}$ excited states. These two methods of finding $\beta$ are compared in our earlier work [14]. In Fig. 1 we show the dependence of strong decay widths of few decay channels on the value of SHO parameter $\beta$. Disk and rectangular marks on each curve correspond to the $\beta$ values obtained by fit to numerically calculated wave functions and to rms radii respectively. These plots show that fitted $\beta$ values lie in the sensitive regions of the curves, which implies that an accurate method of determining the values of the $\beta$ parameter can significantly improve the results.

To calculate the decay rate of a process $A \rightarrow B+C$, we evaluate the matrix element $\left\langle B C\left|H_{I}\right| A\right\rangle$ by using the interaction Hamiltonian of Eq. (7). In general, two different diagrams, shown in Fig. 2, contribute to the matrix element $\left\langle B C\left|H_{I}\right| A\right\rangle$. Using the relativistic phase space factor from Ref. [31] and performing the angular integration gives

$$
\Gamma_{A \rightarrow B C}=2 \pi \frac{P E_{B} E_{C}}{M_{A}} \sum_{L S}\left|\mathcal{M}_{L S}\right|^{2},
$$

where $P=\left|\mathbf{P}_{B}\right|=\left|\mathbf{P}_{C}\right|, M_{A}$ is the mass of the initial meson, and $E_{B}=\sqrt{M_{B}^{2}+P^{2}}$ and $E_{C}=\sqrt{M_{C}^{2}+P^{2}}$ are the energies of the final mesons $B$ and $C$ respectively. Where available, we use experimental masses [16] of $B_{c}$ mesons; otherwise we use the theoretical masses given in Tables I and II. The masses of the final state mesons $D$,
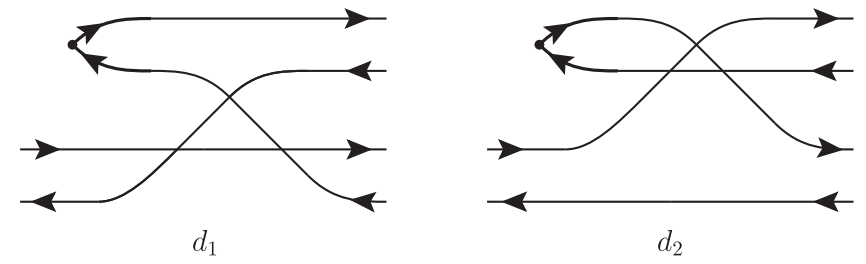

FIG. 2. Decay diagrams in the ${ }^{3} P_{0}$ model. 
TABLE III. Masses and SHO parameter $(\beta)$ for open-charm and open-bottom mesons used in our strong decay width calculations. The experimental masses are taken from PDG [16]. The SHO $\beta$ values are listed in the second column which we obtain by the fit of SHO wave function to the numerical solutions of Schrödinger equation.

\begin{tabular}{|c|c|c|c|}
\hline Meson & $\beta(\mathrm{GeV})$ & $\begin{array}{l}\text { Expt. Mass [16] } \\
(\mathrm{MeV})\end{array}$ & $\begin{array}{c}\text { Mass used in } \\
\text { calculations }(\mathrm{GeV})\end{array}$ \\
\hline$D$ & 0.442 & $\begin{array}{l}D^{ \pm}=1869.58 \pm 0.09 \\
D^{0}=1864.83 \pm 0.08\end{array}$ & 1.867 \\
\hline$D^{*}$ & 0.338 & $\begin{array}{l}D^{* \pm}=2010.26 \pm 0.05 \\
D^{* 0}=2006.85 \pm 0.05\end{array}$ & 2.007 \\
\hline$D_{s}$ & 0.463 & $D_{s}^{ \pm}=1968.27 \pm 0.10$ & 1.968 \\
\hline$D_{s}^{*}$ & 0.369 & $D_{s}^{* \pm}=2112.1 \pm 0.4$ & 2.112 \\
\hline$B$ & 0.405 & $\begin{array}{l}B^{ \pm}=5279.31 \pm 0.15 \\
B^{0}=5279.62 \pm 0.15\end{array}$ & 5.279 \\
\hline$B^{*}$ & 0.372 & $B^{*}=5324.65 \pm 0.25$ & 5.325 \\
\hline$B_{s}$ & 0.429 & $B_{s}^{0}=5366.82 \pm 0.22$ & 5.367 \\
\hline$B_{s}^{*}$ & 0.401 & $B_{s}^{* 0}=5415.4_{-15}^{+1.8}$ & 5.415 \\
\hline
\end{tabular}

$D_{s}, B$, and $B_{s}$ are reported in Table III. The detailed formulism to calculate the matrix element $\left\langle B C\left|H_{I}\right| A\right\rangle$ and decay amplitude $\mathcal{M}_{L S}$ is described in our earlier work [14].

\section{RADIATIVE TRANSITIONS OF $B_{c}$ MESONS}

\section{A. $E 1$ radiative transitions}

$E 1$ radiative partial widths are computed with the following expression [32]:

$$
\begin{aligned}
& \Gamma\left(n^{2 S+1} L_{J} \rightarrow n^{\prime 2 S^{\prime}+1} L_{J^{\prime}}^{\prime}+\gamma\right) \\
& \quad=\frac{4}{3}\left\langle e_{Q}\right\rangle^{2} \alpha \omega^{3} C_{f i} \delta_{S S^{\prime}}\left|\left\langle n^{\prime 2 S^{\prime}+1} L_{J^{\prime}}^{\prime}|r| n^{2 S+1} L_{J}\right\rangle\right|^{2} \frac{E_{f}}{M_{i}},
\end{aligned}
$$

where

$$
\left\langle e_{Q}\right\rangle=\frac{m_{b} e_{c}-m_{c} e_{\bar{b}}}{m_{b}+m_{c}}
$$

Here $e_{c}$ and $e_{\bar{b}}$ are the electric charges of the charm quark and bottom antiquark in units of $|e|, m_{b}$ and $m_{c}$ are the constituent masses of the charm and bottom quarks, $\alpha$ is the fine structure constant, $\omega$ is the final photon energy, $M_{i}$ is mass of the initial meson, and $E_{f}$ is the energy of the final state. Finally, the angular matrix element $C_{f i}$ is given by

$$
C_{f i}=\max \left(L, L^{\prime}\right)\left(2 J^{\prime}+1\right)\left\{\begin{array}{ccc}
L^{\prime} & J^{\prime} & S \\
J & L & 1
\end{array}\right\}^{2} .
$$

Equation (10) is the result of Ref. [32] except for our inclusion of the relativistic phase space factor $E_{f} / M_{i}$ from Refs. [13,27]. The matrix elements $\left\langle n^{\prime 2 S^{\prime}+1} L_{J^{\prime}}^{\prime}|r| n^{2 S+1} L_{J}\right\rangle$ are obtained using the quark model wave functions obtained in Sec. II. Wave function corrections due to perturbative spin-dependent interactions were neglected in this computation, as in Refs. [13,27]. Results for $E 1$ radiative transitions for $B_{c}$ mesons are given in Tables VII-XIV along with the matrix elements so that an interested reader can reproduce our results.

\section{B. $M 1$ radiative transitions}

The $M 1$ radiative partial widths are evaluated using the following expression [33]:

$$
\begin{aligned}
\Gamma\left(n^{2 S+1} L_{J} \rightarrow n^{\prime 2 S^{\prime}+1} L_{J^{\prime}}+\gamma\right) \\
=\frac{\alpha}{3} \omega^{3}\left(2 J^{\prime}+1\right) \delta_{S, S^{\prime} \pm 1} \mid \frac{e_{c}}{m_{c}}\left\langle f\left|j_{0}\left(\frac{m_{b}}{m_{c}+m_{b}} \omega r\right)\right| i\right\rangle \\
\quad-\left.\frac{e_{\bar{b}}}{m_{b}}\left\langle f\left|j_{0}\left(\frac{m_{c}}{m_{c}+m_{b}} \omega r\right)\right| i\right\rangle\right|^{2},
\end{aligned}
$$

where $j_{0}(x)$ is a spherical Bessel function. The definitions of the other parameters are the same as in the $E 1$ radiative transitions. The results for $M 1$ radiative transitions for $B_{c}$ mesons are given in Tables VII-XIV.

\section{E1-E1 HADRONIC TRANSITIONS}

Hadronic transitions are needed to estimate branching ratios and the event rates of decay chains of $B_{c}$ states lying below the $B D$ threshold. The differential rate for $E 1-E 1$ hadronic transitions from an initial meson state $\Phi^{\prime}$ to the final meson state $\Phi$ and a system of light hadrons $h$ is given by $[34,35]$

$$
\frac{d \Gamma}{d \mathcal{M}^{2}}\left[\Phi^{\prime} \rightarrow \Phi+h\right]=(2 J+1) \sum_{k=0}^{2}\left\{\begin{array}{lll}
k & l^{\prime} & l \\
s & J & J^{\prime}
\end{array}\right\}^{2} A_{k}\left(l^{\prime}, l\right),
$$

where $J^{\prime}, J$ are the total angular momentum and $l^{\prime}, l$ are the orbital angular momentum of initial and final meson states respectively, $\{\cdots \ldots\}$ is a 6-j symbol, $\mathcal{M}^{2}$ is the invariant mass squared of the light hadron system, and $A_{k}\left(l^{\prime}, l\right)$ are the reduced matrix elements [34]. Here we use scaling argument to predict the hadronic rates for $c \bar{b}$ mesons using measured rates of $c \bar{c}$ and/or $b \bar{b}$ as input. When measured rates are not available, we use predicted rates of hadronic transition of $b \bar{b}$ states. The scaling law for $E 1-E 1$ hadronic transitions is given by [26,34]

$$
\frac{\Gamma(c \bar{b})}{\Gamma(Q \bar{Q})}=\frac{\left\langle r^{2}(c \bar{b})\right\rangle^{2}}{\left\langle r^{2}(Q \bar{Q})\right\rangle^{2}} p,
$$


TABLE IV. Estimates of reduced rates of hadronic transitions between $c \bar{b}$ mesons.

\begin{tabular}{lcccc}
\hline \hline Transition & $(Q \bar{Q}):$ rate $(\mathrm{keV})$ & $\left\langle r^{2}(c \bar{b})\right\rangle /\left\langle r^{2}(Q \bar{Q})\right\rangle$ & Our reduced $c \bar{b}$ rate $(\mathrm{keV})$ & Reduced $c \bar{b}$ rate $[8](\mathrm{keV})$ \\
\hline $2^{3} S_{1} \rightarrow 1^{3} S_{1}+\pi \pi$ & $(c \bar{c}): 155.84 \pm 5.2^{\mathrm{a}}$ & 0.94 & $A_{0}(0,0)=61.59 \pm 2.1$ & $A_{0}(0,0)=82 \pm 8$ \\
& $(b \bar{b}): 8.46 \pm 0.7^{\mathrm{a}}$ & 2.36 & $A_{0}(0,0)=26.06 \pm 2.2$ & $A_{0}(0,0)=33 \pm 5$ \\
& average: & & $43.83 \pm 2.2$ & $57 \pm 7$ \\
$3^{3} S_{1} \rightarrow 1^{3} S_{1}+\pi \pi$ & $(b \bar{b}): 1.34 \pm 0.12^{\mathrm{a}}$ & 2.24 & $A_{0}^{\prime \prime}(0,0)=2.08 \pm 0.19$ & $A_{0}^{\prime \prime}(0,0)=4.2 \pm 0.6$ \\
$2^{3} P_{0} \rightarrow 1^{3} P_{0}+\pi \pi$ & $(b \bar{b}): 0.44^{\mathrm{b}}$ & 2.23 & $A_{0}(1,1)=1.82$ & $A_{0}(1,1)=2.92$ \\
$2^{3} P_{2} \rightarrow 1^{3} P_{1}+\pi \pi$ & $(b \bar{b}): 0.23^{\mathrm{b}}$ & 2.22 & $A_{2}(1,1)=0.328$ & $A_{2}(1,1)=0.164$ \\
$1^{3} D_{1} \rightarrow 1^{3} S_{1}+\pi \pi$ & $(b \bar{b}): 0.14^{\mathrm{b}}$ & 2.23 & $A_{2}(2,0)=1.183$ & $A_{2}(2,0)=21$ \\
$1^{3} F_{2} \rightarrow 1^{3} P_{0}+\pi \pi$ & $(b \bar{b}): 1.8 \times 10^{-3 \mathrm{~b}}$ & 2.19 & $A_{2}(3,1)=0.114$ & $\cdots$ \\
\hline \hline
\end{tabular}

${ }^{\mathrm{a}}$ From PDG Ref. [16].

${ }^{\mathrm{b}}$ From Ref. [26].

where $\left\langle r^{2}(Q \bar{Q})\right\rangle$ is the expectation value of the square of the interquark separation and $p$ is the phase space factor depending on the masses of initial and final states. The phase space factors for $A_{0}$ and $A_{2}$ reduced matrix elements are $\frac{G\left(\Phi^{\prime}(c \bar{b}) \rightarrow \Phi(c \bar{b}) \pi \pi\right)}{G\left(\Phi^{\prime}(Q \bar{Q}) \rightarrow \Phi(Q \bar{Q}) \pi \pi\right)}$ and $\frac{H\left(\Phi^{\prime}(c \bar{b}) \rightarrow \Phi(c \bar{b}) \pi \pi\right)}{H\left(\Phi^{\prime}(Q \bar{Q}) \rightarrow \Phi(Q \bar{Q}) \pi \pi\right)}$ respectively, where $G$ and $H$ are defined in Ref. [35].

For the $B_{c}(2 S) \rightarrow B_{c}(1 S)+\pi \pi$ reduced rates, we rescale the measured reduced rates of $\psi\left(2^{3} S_{1}\right) \rightarrow \psi\left(1^{3} S_{1}\right)+\pi \pi$ and $\Upsilon\left(2^{3} S_{1}\right) \rightarrow \Upsilon\left(1^{3} S_{1}\right)+\pi \pi$ [16] and take their average value. The reduced rates for $B_{c}(3 S) \rightarrow B_{c}(1 S)+\pi \pi$ are obtained by rescaling the corresponding measured reduced rates of $b \bar{b}$ [16]. There are 16 possible $B_{c}(2 P) \rightarrow$ $B_{c}(1 P)+\pi \pi$ hadronic transitions, which can be expressed in terms of three reduced rates $A_{0}(1,1), A_{1}(1,1)$, and $A_{2}(1,1)$ using Eq. (13). In the soft-pion limit $A_{1}\left(l^{\prime}, l\right)$ contributions are suppressed, so we take $A_{1}(1,1)=0$. $A_{0}(1,1)$ and $A_{2}(1,1)$ are obtained by rescaling the reduced rates of $\Upsilon\left(2^{3} P_{0}\right) \rightarrow \Upsilon\left(1^{3} P_{0}\right)+\pi \pi$ and $\Upsilon\left(2^{3} P_{2}\right) \rightarrow$ $\Upsilon\left(1^{3} P_{1}\right)+\pi \pi$ predicted by Godfrey and Moats [26]. For calculating the hadronic transitions for $B_{c}(1 D) \rightarrow$ $B_{c}(1 S)+\pi \pi$ and $B_{c}(1 F) \rightarrow B_{c}(1 P)+\pi \pi$, we rescale the reduced rates of $\Upsilon\left(1^{3} D_{1}\right) \rightarrow \Upsilon\left(1^{3} S_{1}\right)+\pi \pi$ and $\Upsilon\left(1^{3} F_{2}\right) \rightarrow$ $\Upsilon\left(1^{3} P_{0}\right)+\pi \pi$ which are also taken from Ref. [26]. The scaling factors and reduced rates between $c \bar{b}$ mesons are given in Table IV. These reduced rates are used to determine the $c \bar{b}$ hadronic transitions which are

TABLE V. Rates for the $E 1-E 1$ hadronic transitions of $2 S, 3 S$, and $2 P$ states of $B_{c}$ mesons.

\begin{tabular}{|c|c|c|}
\hline Transition & Expression for the rate & the $c \bar{b}$ rate $(\mathrm{keV})$ \\
\hline $2^{1} S_{0} \rightarrow 1^{1} S_{0}+\pi \pi$ & $A_{0}(0,0)$ & 10.68 \\
\hline $2{ }^{3} S_{1} \rightarrow 1{ }^{3} S_{1}+\pi \pi$ & $A_{0}(0,0)$ & 3.99 \\
\hline $3{ }^{1} S_{0} \rightarrow{ }^{1} S_{0}+\pi \pi$ & $A_{0}^{\prime \prime}(0,0)$ & 0.97 \\
\hline $3^{3} S_{1} \rightarrow 1^{3} S_{1}+\pi \pi$ & $A_{0}^{\prime \prime}(0,0)$ & 0.81 \\
\hline $2^{3} P_{0} \rightarrow 1 P_{1}+\pi \pi$ & $\frac{1}{3} A_{1}(1,1)$ & 0 \\
\hline $2^{3} P_{0} \rightarrow 1 P_{1}^{\prime}+\pi \pi$ & $\frac{1}{3} A_{1}(1,1)$ & 0 \\
\hline $2^{3} P_{0} \rightarrow 1^{3} P_{0}+\pi \pi$ & $\frac{1}{3} A_{0}(1,1)$ & 0.002 \\
\hline $2^{3} P_{2} \rightarrow 1^{3} P_{2}+\pi \pi$ & $\frac{1}{3} A_{0}(1,1)+\frac{1}{4} A_{1}(1,1)+\frac{7}{60} A_{2}(1,1)$ & 0.0007 \\
\hline $2^{3} P_{2} \rightarrow 1 P_{1}+\pi \pi$ & $\frac{1}{12} A_{1}(1,1)+\frac{3}{20} A_{2}(1,1)$ & 0.0002 \\
\hline $2^{3} P_{2} \rightarrow 1 P_{1}^{\prime}+\pi \pi$ & $\frac{1}{12} A_{1}(1,1)+\frac{3}{20} A_{2}(1,1)$ & 0.0002 \\
\hline $2^{3} P_{2} \rightarrow 1^{3} P_{0}+\pi \pi$ & $\frac{1}{15} A_{2}(1,1)$ & 0.0005 \\
\hline $2 P_{1} \rightarrow 1 P_{1}+\pi \pi$ & $\frac{1}{3} A_{0}(1,1)+\frac{1}{12} A_{1}(1,1)+\frac{1}{12} A_{2}(1,1)^{\mathrm{a}}$ & 0.0003 \\
\hline $2 P_{1} \rightarrow 1^{3} P_{0}+\pi \pi$ & $\frac{1}{9} A_{1}(1,1)$ & 0 \\
\hline $2 P_{1}^{\prime} \rightarrow 1 P_{1}+\pi \pi$ & $\frac{1}{3} A_{0}(1,1)+\frac{1}{3} A_{1}(1,1)+\frac{1}{3} A_{2}(1,1)^{\mathrm{b}}$ & $3 \times 10^{-6}$ \\
\hline $2 P_{1}^{\prime} \rightarrow 1 P_{1}^{\prime}+\pi \pi$ & $\frac{1}{3} A_{0}(1,1)+\frac{1}{3} A_{1}(1,1)+\frac{1}{3} A_{2}(1,1)^{\mathrm{b}}$ & 0.0014 \\
\hline $2 P_{1}^{\prime} \rightarrow 1^{3} P_{0}+\pi \pi$ & $\frac{1}{9} A_{1}(1,1)$ & 0 \\
\hline
\end{tabular}

${ }^{\mathrm{a}}$ The expression is for ${ }^{3} P_{1} \rightarrow{ }^{3} P_{1}$ transition.

${ }^{\mathrm{b}}$ The expression is for ${ }^{1} P_{1} \rightarrow{ }^{1} P_{1}$ transition. 
TABLE VI. Rates for the $E 1-E 1$ hadronic transitions of $1 D$ and $1 F$ states of $B_{c}$ mesons.

\begin{tabular}{lcc}
\hline \hline Transition & Expression for the rate & the $c \bar{b}$ rate $(\mathrm{keV})$ \\
\hline $1^{3} D_{1} \rightarrow 1^{3} S_{1}+\pi \pi$ & $\frac{1}{5} A_{2}(2,0)$ & 0.042 \\
$1^{3} D_{3} \rightarrow 1^{3} S_{1}+\pi \pi$ & $\frac{1}{5} A_{2}(2,0)$ & 0.043 \\
$1 D_{2} \rightarrow 1^{1} S_{0}+\pi \pi$ & $\frac{1}{5} A_{2}(2,0)$ & 0.035 \\
$1 D_{2} \rightarrow 1^{3} S_{1}+\pi \pi$ & $\frac{1}{5} A_{2}(2,0)$ & 0.017 \\
$1 D_{2}^{\prime} \rightarrow 1^{1} S_{0}+\pi \pi$ & $\frac{1}{5} A_{2}(2,0)$ & 0.23 \\
$1 D_{2}^{\prime} \rightarrow 1^{3} S_{1}+\pi \pi$ & $\frac{1}{5} A_{2}(2,0)$ & 0.026 \\
$1^{3} F_{2} \rightarrow 1^{3} P_{0}+\pi \pi$ & $\frac{1}{15} A_{2}(3,1)$ & 0.0004 \\
$1^{3} F_{2} \rightarrow 1^{3} P_{2}+\pi \pi$ & $\frac{1}{105} A_{2}(3,1)$ & 0.00004 \\
$1^{3} F_{2} \rightarrow 1 P_{1}+\pi \pi$ & $\frac{1}{15} A_{2}(3,1)$ & 0.0001 \\
$1^{3} F_{2} \rightarrow 1 P_{1}^{\prime}+\pi \pi$ & $\frac{1}{15} A_{2}(3,1)$ & 0.0002 \\
$1 F_{3} \rightarrow 1^{3} P_{2}+\pi \pi$ & $\frac{1}{21} A_{2}(3,1)$ & 0.0001 \\
$1 F_{3} \rightarrow 1 P_{1}+\pi \pi$ & $\frac{2}{21} A_{2}(3,1)^{\mathrm{a}}$ & 0.001 \\
$1 F_{3} \rightarrow 1 P_{1}^{\prime}+\pi \pi$ & $\frac{2}{21} A_{2}(3,1)^{\mathrm{a}}$ & $\sim 0$ \\
$1 F_{3}^{\prime} \rightarrow 1^{3} P_{2}+\pi \pi$ & $\frac{1}{21} A_{2}(3,1)$ & 0.0001 \\
$1 F_{3}^{\prime} \rightarrow 1 P_{1}+\pi \pi$ & $\frac{1}{7} A_{2}(3,1)^{\mathrm{b}}$ & 0.00002 \\
$1 F_{3}^{\prime} \rightarrow 1 P_{1}^{\prime}+\pi \pi$ & $\frac{1}{7} A_{2}(3,1)^{\mathrm{b}}$ & 0.001 \\
$1^{3} F_{4} \rightarrow 1^{3} P_{2}+\pi \pi$ & $\frac{1}{7} A_{2}(3,1)$ & 0.0005 \\
\hline \hline
\end{tabular}

${ }^{\mathrm{a}}$ The expression is for ${ }^{3} F_{3} \rightarrow{ }^{3} P_{1}$ transition.

${ }^{\mathrm{b}}$ The expression is for ${ }^{1} F_{3} \rightarrow{ }^{1} P_{1}$ transition.

summarized in Tables V and VI. To calculate the transition rates for mixed states, mixing angles are incorporated, with the values taken from Tables I and II.

In Tables VII-XIV, we combine the widths of radiative decays, strong decays, and hadronic transitions to calculate the total widths and the branching ratios. These BR's are used in the next section to give estimates for the number of events expected at the LHC for different decay chains of $B_{c}$ states below the threshold. In these tables $c_{P}=\cos \phi_{n P}$, $s_{P}=\sin \phi_{n P}, c_{D}=\cos \phi_{n D}, s_{D}=\sin \phi_{n D}, c_{F}=\cos \phi_{n F}$, and $s_{F}=\sin \phi_{n F}$, with $n$ being the principal quantum number.

\section{EXPERIMENTAL SIGNATURES AND SEARCH STRATEGIES}

Our calculated masses of $B_{c}$ states show that there are three $S$-wave, two $P$-wave, two $D$-wave, and one $F$-wave $B_{c}$ multiplets lying below the $B D$ threshold $(\approx 7144) \mathrm{MeV}$. These are the narrow states of the $B_{c}$ spectrum because $B_{c}$ cannot annihilate into gluons due to its nonzero flavor. All these excited states below the $B D$ threshold will cascade decay into the ground state $B_{c}$ through emission of photons and/or pions, which eventually decays through weak interaction. These photons and pions produced by electromagnetic and/or hadronic transitions carry unique signature of initial $B_{c}$. Hence the production events of $B_{c}$ excited states can be reconstructed by detecting and measuring the energies of produced photons and pions. On the other hand the excited states above the $B D$ threshold will rapidly decay into a pair of $B\left(B_{s}\right)$ and $D\left(D_{s}\right)$ mesons through strong interaction processes. In order to predict observable event rates of $B_{c}$ excited states (below $B D$ threshold) in $p p$ collision at the LHC, we require the knowledge of their production cross sections, branching ratios of their electromagnetic and hadronic transitions, and the branching ratios of weak decay channels of $B_{c}$ ground states through which its production can be identified experimentally.

Inclusive production cross sections of $B_{c}$ states in $p p$ collision at LHC energy has been calculated in Refs. [36-39] using the fragmentation approach and in Refs. [40-45] using the pQCD approach. The results of the two approaches quantitatively agree for $p_{T} \geq 10 \mathrm{GeV}$ [44].

The fragmentation approach of Refs. [36,37] shows that production cross sections of $B_{c}\left(1^{1} S_{0}\right)$ and $B_{c}\left(1^{3} S_{1}\right)$ states are 0.72 and $1.21 \mathrm{nb}$ respectively for transverse momentum $p_{T}\left(B_{c}\right)>20 \mathrm{GeV}$ and rapidity $\left|y\left(B_{c}\right)\right|<2.5$ at LHC. These values include the contribution of both $\bar{b}$-quark and gluon fragmentation functions of the $B_{c}$ states. When these values are extrapolated to kinematic cut $p_{T}\left(B_{c}\right)>$ $10 \mathrm{GeV}$ using the values reported in Table III of Ref. [38], we obtain the production cross sections 5.5 and $9.3 \mathrm{nb}$ for the $1^{1} S_{0}$ and $1^{3} S_{1}$ states respectively. The production cross sections of the $2^{1} S_{0}$ and $2^{3} S_{1}$ states are obtained by multiplying the corresponding values of $1 S$ states with the factor $\left|R_{2 S}(0) / R_{1 S}(0)\right|^{2} \simeq 0.6$ [36]. In Ref. [38] production cross sections of $1 P$ and $2 P$ states are calculated using the fragmentation approach. However, for the LHC they report only the total cross section that includes the contribution from $1 S, 2 S, 1 P$, and $2 P$ states. The reported value $33.8 \mathrm{nb}$ for kinematic cuts $p_{T}\left(B_{c}\right)>10 \mathrm{GeV}$ and $\left|y\left(B_{c}\right)\right|<2.5$ implies that total contribution of $1 P$ and $2 P$ states is $10.2 \mathrm{nb}$. We divide this value over eight $1 P$ and $2 P$ states using the distribution reported for Tevatron in Figs. 3 and 4 of Ref. [38]. It is pointed out in Ref. [37] that the distribution is not much changed at the LHC energy. In Ref. [39], it is shown that total fragmentation probability for a $\bar{b}$-quark to produce the $\mathrm{D}$-wave $B_{c}$ mesons is about $2 \times 10^{-5}$, equivalent to $2 \%$ of the total inclusive cross section of all of $B_{c}$ states lying below the $B D$ threshold. These estimates of the cross sections are used to predict the number of events of various decay chains of excited $B_{c}$ states.

The excited states below the $B D$ threshold eventually decay into the $B_{c}$ ground state; therefore, it is important to observe this state in order to reconstruct the events of originally produced states. Prominent weak decay modes of $B_{c}$ ground states are summarized in Table 10 of Ref. [8]. We assume that ground state $B_{c}$ is observed through two golden channels: (i) $B_{c}^{ \pm} \rightarrow J / \psi+\pi^{ \pm} \rightarrow l^{-} l^{+} \pi^{ \pm}$ having combined BR of $0.013 \%$ and detection efficiency 
TABLE VII. Partial widths and branching ratios for strong, radiative, and hadronic transitions for the $1 S, 2 S, 3 S$, and $4 S$ states of $B_{c}$ mesons. Column 4 labeled $\mathcal{M}$ gives the matrix element appropriate to the particular decay. For $E 1$ and $M 1$ transitions matrix elements are $\left\langle\psi_{f}|r| \psi_{i}\right\rangle$ and $\left\langle\psi_{f}\left|j_{0}\left(k r \frac{m_{b, c}}{m_{c}+m_{b}}\right)\right| \psi_{i}\right\rangle$ are in units of $\mathrm{GeV}^{-1}$ and the strong decay amplitudes are in units of $\mathrm{GeV}^{-1 / 2}$. Details of the calculations are given in the text.

\begin{tabular}{|c|c|c|c|c|c|}
\hline Meson & Decay mode & Photon energy $\mathrm{MeV}$ & Amplitude $(\mathcal{M})$ & $\Gamma_{\text {thy }} \mathrm{MeV}$ & B.R $(\%)$ \\
\hline$\overline{B_{c}^{*}}$ & $B_{c} \gamma$ & 60.81 & $\left\langle 1^{1} S_{0}\left|j_{0}\left(k r \frac{m_{b, c}}{m_{c}+m_{b}}\right)\right| 1^{3} S_{1}\right\rangle=0.9983,0.999$ & $0.15 \mathrm{keV}$ & 100 \\
\hline \multirow{5}{*}{$B_{c}\left(2^{1} S_{0}\right)$} & $B_{c}\left(1^{1} S_{0}\right)+\pi \pi$ & & & $10.68 \mathrm{keV}$ & 51.42 \\
\hline & $B_{c}^{*} \gamma$ & 392.83 & $\left\langle 1^{3} S_{1}\left|j_{0}\left(k r \frac{m_{b, c}}{m_{c}+m_{b}}\right)\right| 2^{1} S_{0}\right\rangle=0.0316,-0.0182$ & $0.07 \mathrm{keV}$ & 0.34 \\
\hline & $B_{c}\left(1 P_{1}\right) \gamma$ & 90.39 & $\left\langle 1^{1} P_{1}|r| 2^{1} S_{0}\right\rangle=-2.8218$ & $7.11 \mathrm{keV}$ & 34.23 \\
\hline & $B_{c}\left(1 P_{1}^{\prime}\right) \gamma$ & 84.46 & $\left\langle 1^{1} P_{1}|r| 2^{1} S_{0}\right\rangle=-2.8218$ & $2.91 \mathrm{keV}$ & 14.01 \\
\hline & total & & & $20.77 \mathrm{keV}$ & 100 \\
\hline \multirow[t]{8}{*}{$B_{c}\left(2^{3} S_{1}\right)$} & $B_{c}\left(1^{3} S_{1}\right)+\pi \pi$ & & & $3.99 \mathrm{keV}$ & 26.76 \\
\hline & $B_{c} \gamma$ & 455.58 & $\left\langle 1^{1} S_{0}\left|j_{0}\left(k r \frac{m_{b, c}}{m_{c}+m_{b}}\right)\right| 2^{3} S_{1}\right\rangle=0.0887,0.0303$ & $0.41 \mathrm{keV}$ & 2.75 \\
\hline & $B_{c}\left(2^{1} S_{0}\right) \gamma$ & 6.0 & $\left\langle 2^{1} S_{0}\left|j_{0}\left(k r \frac{m_{c, c}}{m_{c}+m_{b}}\right)\right| 2^{3} S_{1}\right\rangle=0.9994,0.9995$ & $1.4 \times 10^{-4} \mathrm{keV}$ & 0.001 \\
\hline & $B_{c}\left(1^{3} P_{2}\right) \gamma$ & 81.5 & $\left\langle 1^{3} P_{2}|r| 2^{3} S_{1}\right\rangle=-2.8099$ & $4.31 \mathrm{keV}$ & 28.91 \\
\hline & $B_{c}\left(1 P_{1}\right) \gamma$ & 96.3 & $\left\langle 1^{3} P_{1}|r| 2^{3} S_{1}\right\rangle=-2.8099$ & $1.43 \mathrm{keV}$ & 9.59 \\
\hline & $B_{c}\left(1 P_{1}^{\prime}\right) \gamma$ & 90.39 & $\left\langle 1^{3} P_{1}|r| 2^{3} S_{1}\right\rangle=-2.8099$ & $2.35 \mathrm{keV}$ & 15.76 \\
\hline & $B_{c}\left(1^{3} P_{0}\right) \gamma$ & 115.0 & $\left\langle 1^{3} P_{0}|r| 2^{3} S_{1}\right\rangle=-2.8099$ & $2.42 \mathrm{keV}$ & 16.23 \\
\hline & total & & & $14.91 \mathrm{keV}$ & 100 \\
\hline \multirow[t]{8}{*}{$B_{c}\left(3^{1} S_{0}\right)$} & $B_{c}\left(1^{1} S_{0}\right)+\pi \pi$ & & & $0.97 \mathrm{keV}$ & 4.71 \\
\hline & $B_{c}^{*} \gamma$ & 645.23 & $\left\langle 1^{3} S_{1}\left|j_{0}\left(k r \frac{m_{b, c}}{m_{c}+m_{b}}\right)\right| 3^{1} S_{0}\right\rangle=0.0259,-0.0079$ & $0.24 \mathrm{keV}$ & 1.17 \\
\hline & $B_{c}\left(2^{3} S_{1}\right) \gamma$ & 261.92 & $\left\langle 2^{3} S_{1}\left|j_{0}\left(k r \frac{m_{b, c}}{m_{c}+m_{b}}\right)\right| 3^{1} S_{0}\right\rangle=0.0546,-0.0101$ & $0.07 \mathrm{keV}$ & 0.34 \\
\hline & $B_{c}\left(1 P_{1}\right) \gamma$ & 354.55 & $\left\langle 1^{3} P_{1}|r| 3^{1} S_{0}\right\rangle=0.1055$ & $0.6 \mathrm{keV}$ & 2.92 \\
\hline & $B_{c}\left(1 P_{1}^{\prime}\right) \gamma$ & 348.86 & $\left\langle 1^{3} P_{1}|r| 3^{1} S_{0}\right\rangle=0.1055$ & $0.29 \mathrm{keV}$ & 1.41 \\
\hline & $B_{c}\left(2 P_{1}\right) \gamma$ & 83.50 & $\left\langle 2^{3} P_{1}|r| 3^{1} S_{0}\right\rangle=-4.3884$ & $13.56 \mathrm{keV}$ & 65.89 \\
\hline & $B_{c}\left(2 P_{1}^{\prime}\right) \gamma$ & 74.60 & $\left\langle 2^{3} P_{1}|r| 3^{1} S_{0}\right\rangle=-4.3884$ & $4.85 \mathrm{keV}$ & 23.57 \\
\hline & total & & & $20.58 \mathrm{keV}$ & 100 \\
\hline \multirow[t]{13}{*}{$B_{c}\left(3^{3} S_{1}\right)$} & $B_{c}\left(1^{3} S_{1}\right)+\pi \pi$ & & & $0.81 \mathrm{keV}$ & 4.12 \\
\hline & $B_{c} \gamma$ & 703.76 & $\left\langle 1^{1} S_{0}\left|j_{0}\left(k r \frac{m_{b, c}}{m_{c}+m_{b}}\right)\right| 3^{3} S_{1}\right\rangle=0.0552,0.0145$ & $0.57 \mathrm{keV}$ & 2.9 \\
\hline & $B_{c}\left(2^{1} S_{0}\right) \gamma$ & 271.53 & $\left\langle 2^{1} S_{0}\left|j_{0}\left(k r \frac{m_{b, c}}{m_{c}+m_{b}}\right)\right| 3^{3} S_{1}\right\rangle=0.0889,0.0246$ & $0.08 \mathrm{keV}$ & 0.41 \\
\hline & $B_{c}\left(3^{1} S_{0}\right) \gamma$ & 4.0 & $\left\langle 3^{1} S_{0}\left|j_{0}\left(k r \frac{m_{b, c}}{m_{c}+m_{b}}\right)\right| 3^{3} S_{1}\right\rangle=0.9996,0.9996$ & $4.2 \times 10^{-5} \mathrm{keV}$ & 0.0002 \\
\hline & $B_{c}\left(1^{3} P_{2}\right) \gamma$ & 344.12 & $\left\langle 1^{3} P_{2}|r| 3^{3} S_{1}\right\rangle=0.0926$ & $0.35 \mathrm{keV}$ & 1.78 \\
\hline & $B_{c}\left(1 P_{1}\right) \gamma$ & 358.35 & $\left\langle 1^{3} P_{1}|r| 3^{3} S_{1}\right\rangle=0.0926$ & $0.08 \mathrm{keV}$ & 0.41 \\
\hline & $B_{c}\left(1 P_{1}^{\prime}\right) \gamma$ & 352.66 & $\left\langle 1^{3} P_{1}|r| 3^{3} S_{1}\right\rangle=0.0926$ & $0.15 \mathrm{keV}$ & 0.76 \\
\hline & $B_{c}\left(1^{3} P_{0}\right) \gamma$ & 376.33 & $\left\langle 1^{3} P_{0}|r| 3^{3} S_{1}\right\rangle=0.0926$ & $0.09 \mathrm{keV}$ & 0.46 \\
\hline & $B_{c}\left(2^{3} P_{2}\right) \gamma$ & 71.63 & $\left\langle 2^{3} P_{2}|r| 3^{3} S_{1}\right\rangle=-4.3738$ & $7.09 \mathrm{keV}$ & 36.03 \\
\hline & $B_{c}\left(2 P_{1}\right) \gamma$ & 87.45 & $\left\langle 2^{3} P_{1}|r| 3^{3} S_{1}\right\rangle=-4.3738$ & $2.59 \mathrm{keV}$ & 13.16 \\
\hline & $B_{c}\left(2 P_{1}^{\prime}\right) \gamma$ & 78.56 & $\left\langle 2^{3} P_{1}|r| 3^{3} S_{1}\right\rangle=-4.3738$ & $3.74 \mathrm{keV}$ & 19.0 \\
\hline & $B_{c}\left(2^{3} P_{0}\right) \gamma$ & 102.24 & $\left\langle 2^{3} P_{0}|r| 3^{3} S_{1}\right\rangle=-4.3738$ & $4.13 \mathrm{keV}$ & 20.99 \\
\hline & total & & & $19.68 \mathrm{keV}$ & 100 \\
\hline$B_{c}\left(4^{1} S_{0}\right)$ & $D B^{*}$ & & ${ }^{3} P_{0}=+0.0305$ & 2.96 & 100 \\
\hline \multirow[t]{3}{*}{$B_{c}\left(4^{3} S_{1}\right)$} & $D B$ & & ${ }^{1} P_{1}=-0.0067$ & 0.21 & 11.66 \\
\hline & $D B^{*}$ & & ${ }^{3} P_{1}=-0.022$ & 1.59 & 88.29 \\
\hline & total & & & 1.8 & 100 \\
\hline
\end{tabular}


TABLE VIII. Partial widths and branching ratios for strong, radiative, and hadronic transitions for the $1 P, 2 P$, and $3 P$ states of $B_{c}$ mesons.

\begin{tabular}{|c|c|c|c|c|c|}
\hline Meson & Decay mode & Photon energy $\mathrm{MeV}$ & Amplitude $(\mathcal{M})$ & $\Gamma_{\text {thy }} \mathrm{MeV}$ & B.R $(\%)$ \\
\hline$B_{c}\left(1^{3} P_{0}\right)$ & $B_{c}^{*} \gamma$ & 288.44 & $\left\langle 1^{3} S_{1}|r| 1^{3} P_{0}\right\rangle=1.896$ & $52.23 \mathrm{keV}$ & 100 \\
\hline$B_{c}\left(1 P_{1}\right)$ & $\begin{array}{c}B_{c} \gamma \\
B_{c}^{*} \gamma \\
\text { total }\end{array}$ & $\begin{array}{l}323.71 \\
306.59\end{array}$ & $\begin{array}{c}\left\langle 1^{1} S_{0}|r| 1^{1} P_{1}\right\rangle=1.8362 \\
\left\langle 1^{3} S_{1}|r| 1^{3} P_{1}\right\rangle=1.896\end{array}$ & $\begin{array}{l}46.13 \mathrm{keV} \\
20.94 \mathrm{keV} \\
67.07 \mathrm{keV}\end{array}$ & $\begin{array}{c}68.78 \\
31.22 \\
100\end{array}$ \\
\hline$B_{c}\left(1 P_{1}^{\prime}\right)$ & $\begin{array}{c}B_{c} \gamma \\
B_{c}^{*} \gamma \\
\text { total }\end{array}$ & $\begin{array}{l}329.42 \\
312.31\end{array}$ & $\begin{array}{c}\left\langle 1^{1} S_{0}|r| 1^{1} P_{1}\right\rangle=1.8362 \\
\left\langle 1^{3} S_{1}|r| 1^{3} P_{1}\right\rangle=1.896\end{array}$ & $\begin{array}{l}24.37 \mathrm{keV} \\
44.16 \mathrm{keV} \\
68.53 \mathrm{keV}\end{array}$ & $\begin{array}{c}35.56 \\
64.44 \\
100\end{array}$ \\
\hline$B_{c}\left(1^{3} P_{2}\right)$ & $B_{c}^{*} \gamma$ & 320.88 & $\left\langle 1^{3} S_{1}|r| 1^{3} P_{2}\right\rangle=1.896$ & $71.91 \mathrm{keV}$ & 100 \\
\hline$B_{c}\left(2^{3} P_{0}\right)$ & $\begin{array}{c}B_{c}\left(1^{3} P_{0}\right)+\pi \pi \\
B_{c}^{*} \gamma \\
B_{c}\left(2^{3} S_{1}\right) \gamma \\
B_{c}\left(1^{3} D_{1}\right) \gamma \\
\text { total }\end{array}$ & $\begin{array}{l}554.76 \\
165.96 \\
73.60\end{array}$ & $\begin{array}{c}\left\langle 1^{3} S_{1}|r| 2^{3} P_{0}\right\rangle=0.4117 \\
\left\langle 2^{3} S_{1}|r| 2^{3} P_{0}\right\rangle=3.1266 \\
\left\langle 1^{3} D_{1}|r| 2^{3} P_{0}\right\rangle=-2.9727\end{array}$ & $\begin{array}{c}0.002 \mathrm{keV} \\
17.52 \mathrm{keV} \\
27.05 \mathrm{keV} \\
4.27 \mathrm{keV} \\
48.84 \mathrm{keV}\end{array}$ & $\begin{array}{c}0.004 \\
35.87 \\
55.38 \\
8.74 \\
100\end{array}$ \\
\hline$B_{c}\left(2 P_{1}\right)$ & $\begin{array}{c}B_{c}\left(1 P_{1}\right)+\pi \pi \\
B_{c} \gamma \\
B_{c}^{*} \gamma \\
B_{c}\left(2^{1} S_{0}\right) \gamma \\
B_{c}\left(2^{3} S_{1}\right) \gamma \\
B_{c}\left(1 D_{2}\right) \gamma \\
B_{c}\left(1 D_{2}^{\prime}\right) \gamma \\
\text { total }\end{array}$ & $\begin{array}{c}584.98 \\
568.54 \\
186.42 \\
180.58 \\
84.48 \\
84.48\end{array}$ & $\begin{aligned}\left\langle 1^{1} S_{0}|r| 2^{1} P_{1}\right\rangle & =0.4258 \\
\left\langle 1^{3} S_{1}|r| 2^{3} P_{1}\right\rangle & =0.4117 \\
\left\langle 2^{1} S_{0}|r| 2^{1} P_{1}\right\rangle & =3.0728 \\
\left\langle 2^{3} S_{1}|r| 2^{3} P_{1}\right\rangle & =3.1266 \\
\left\langle 1^{3} D_{2}|r| 2^{3} P_{1}\right\rangle & =-2.9727 \\
\left\langle 1^{1} D_{2}|r| 2^{1} P_{1}\right\rangle & =-2.9929 \\
\left\langle 1^{3} D_{2}|r| 2^{3} P_{1}\right\rangle & =-2.9727 \\
\left\langle 1^{1} D_{2}|r| 2^{1} P_{1}\right\rangle & =-2.9929\end{aligned}$ & $\begin{array}{c}0.0003 \mathrm{keV} \\
14.64 \mathrm{keV} \\
6.3 \mathrm{keV} \\
24.67 \mathrm{keV} \\
11.64 \mathrm{keV} \\
5.86 \mathrm{keV} \\
\\
0.11 \mathrm{keV} \\
\\
63.22 \mathrm{keV}\end{array}$ & $\begin{array}{c}0.0005 \\
23.16 \\
9.97 \\
39.02 \\
18.41 \\
9.27\end{array}$ \\
\hline$B_{c}\left(2 P_{1}^{\prime}\right)$ & $\begin{array}{c}B_{c}\left(1 P_{1}\right)+\pi \pi \\
B_{c}\left(1 P_{1}^{\prime}\right)+\pi \pi \\
B_{c} \gamma \\
B_{c}^{*} \gamma \\
B_{c}\left(2^{1} S_{0}\right) \gamma \\
B_{c}\left(2^{3} S_{1}\right) \gamma \\
B_{c}\left(1^{3} D_{1}\right) \gamma \\
B_{c}\left(1 D_{2}\right) \gamma \\
B_{c}\left(1 D_{2}^{\prime}\right) \gamma \\
\text { total }\end{array}$ & $\begin{array}{c}593.21 \\
576.80 \\
195.18 \\
189.34 \\
97.31 \\
93.36 \\
\\
93.36\end{array}$ & $\begin{aligned}\left\langle 1^{1} S_{0}|r| 2^{1} P_{1}\right\rangle & =0.4258 \\
\left\langle 1^{3} S_{1}|r| 2^{3} P_{1}\right\rangle & =0.4117 \\
\left\langle 2^{1} S_{0}|r| 2^{1} P_{1}\right\rangle & =3.0728 \\
\left\langle 2^{3} S_{1}|r| 2^{3} P_{1}\right\rangle & =3.1266 \\
\left\langle 1^{3} D_{1}|r| 2^{3} P_{1}\right\rangle & =-2.9727 \\
\left\langle 1^{3} D_{2}|r| 2^{3} P_{1}\right\rangle & =-2.9727 \\
\left\langle 1^{1} D_{2}|r| 2^{1} P_{1}\right\rangle & =-2.9929 \\
\left\langle 1^{3} D_{2}|r| 2^{3} P_{1}\right\rangle & =-2.9727 \\
\left\langle 1^{1} D_{2}|r| 2^{1} P_{1}\right\rangle & =-2.9929\end{aligned}$ & $\begin{array}{c}3 \times 10^{-6} \mathrm{keV} \\
0.0014 \mathrm{keV} \\
7.65 \mathrm{keV} \\
13.12 \mathrm{keV} \\
14.19 \mathrm{keV} \\
26.76 \mathrm{keV} \\
1.64 \mathrm{keV} \\
1.5 \times 10^{-4} \mathrm{keV} \\
7.3 \mathrm{keV} \\
70.66 \mathrm{keV}\end{array}$ & $\begin{array}{c}\sim 0 \\
0.002 \\
10.83 \\
18.57 \\
20.08 \\
37.87 \\
2.32 \\
0.0002 \\
\\
10.33 \\
\\
100\end{array}$ \\
\hline$B_{c}\left(2^{3} P_{2}\right)$ & $\begin{array}{c}B_{c}\left(1^{3} P_{2}\right)+\pi \pi \\
B_{c}\left(1 P_{1}\right)+\pi \pi \\
B_{c}\left(1 P_{1}^{\prime}\right)+\pi \pi \\
B_{c}\left(1^{3} P_{0}\right)+\pi \pi \\
B_{c}^{*} \gamma \\
B_{c}\left(2^{3} S_{1}\right) \gamma \\
B_{c}\left(1^{3} D_{1}\right) \gamma \\
B_{c}\left(1^{3} D_{3}\right) \gamma \\
B_{c}\left(1 D_{2}\right) \gamma \\
B_{c}\left(1 D_{2}^{\prime}\right) \gamma \\
\text { total }\end{array}$ & $\begin{array}{c}583.21 \\
196.15 \\
104.21 \\
98.29 \\
100.27 \\
100.27\end{array}$ & $\begin{aligned}\left\langle 1^{3} S_{1}|r| 2^{3} P_{2}\right\rangle & =0.4117 \\
\left\langle 2^{3} S_{1}|r| 2^{3} P_{2}\right\rangle & =3.1266 \\
\left\langle 1^{3} D_{1}|r| 2^{3} P_{2}\right\rangle & =-2.9727 \\
\left\langle 1^{3} D_{3}|r| 2^{3} P_{2}\right\rangle & =-2.9727 \\
\left\langle 1^{3} D_{2}|r| 2^{3} P_{2}\right\rangle & =-2.9727 \\
\left\langle 1^{3} D_{2}|r| 2^{3} P_{2}\right\rangle & =-2.9727\end{aligned}$ & $\begin{array}{c}0.0007 \mathrm{keV} \\
0.0002 \mathrm{keV} \\
0.0002 \mathrm{keV} \\
0.0005 \mathrm{keV} \\
20.36 \mathrm{keV} \\
44.67 \mathrm{keV} \\
0.12 \mathrm{keV} \\
8.54 \mathrm{keV} \\
0.65 \mathrm{keV} \\
0.97 \mathrm{keV} \\
75.31 \mathrm{keV}\end{array}$ & $\begin{array}{c}0.0009 \\
0.0003 \\
0.0003 \\
0.0007 \\
27.03 \\
59.31 \\
0.16 \\
11.34 \\
0.86 \\
1.29 \\
100\end{array}$ \\
\hline$B_{c}\left(3^{3} P_{0}\right)$ & $D B$ & & ${ }^{1} S_{0}=+0.1157$ & 3.34 & 100 \\
\hline$B_{c}\left(3^{3} P_{2}\right)$ & $D B$ & & ${ }^{1} D_{2}=-0.0215$ & 1.16 & 100 \\
\hline
\end{tabular}


TABLE IX. Partial widths and branching ratios for strong, radiative, and hadronic transitions for the $4 P$ states of $B_{c}$ mesons.

\begin{tabular}{|c|c|c|c|c|c|}
\hline Meson & Decay mode & Photon energy $\mathrm{MeV}$ & Amplitude $(\mathcal{M})$ & $\Gamma_{\text {thy }} \mathrm{MeV}$ & B.R \\
\hline \multirow{5}{*}{$\overline{B_{c}\left(4^{3} P_{0}\right)}$} & $D B$ & & ${ }^{1} S_{0}=+0.008$ & 0.45 & 9.03 \\
\hline & $D^{*} B^{*}$ & & ${ }^{1} S_{0}=-0.0196$ & 4.53 & 90.93 \\
\hline & & & ${ }^{5} D_{0}=-0.0429$ & & \\
\hline & $D_{s} B_{s}$ & & ${ }^{1} S_{0}=+0.001$ & 0.002 & 0.04 \\
\hline & total & & & 4.98 & 100 \\
\hline \multirow{6}{*}{$B_{c}\left(4 P_{1}\right)$} & $D B^{*}$ & & ${ }^{3} S_{1}=-0.0083 c_{P}+0.0117 s_{P}$ & 1.03 & 14.21 \\
\hline & & & ${ }^{3} D_{1}=-0.0104 c_{P}-0.0072 s_{P}$ & & \\
\hline & $D^{*} B$ & & ${ }^{3} S_{1}=-0.0127 c_{P}+0.0176 s_{P}$ & 0.94 & 12.97 \\
\hline & $D^{*} B^{*}$ & & $\begin{array}{c}{ }^{3} D_{1}=-0.0120 c_{P}-0.0082 s_{P} \\
{ }^{3} S_{1}=+0.0006 c_{P}\end{array}$ & 5.28 & 72.83 \\
\hline & & & $\begin{array}{c}{ }^{3} D_{1}=+0.0406 c_{P} \\
{ }^{5} D_{1}=-0.0491 s_{P}\end{array}$ & & \\
\hline & total & & & 7.25 & 100 \\
\hline \multirow[t]{6}{*}{$B_{c}\left(4 P_{1}^{\prime}\right)$} & $D B^{*}$ & & ${ }^{3} S_{1}=+0.0120 c_{P}+0.0086 s_{P}$ & 1.44 & 13.81 \\
\hline & & & $\begin{array}{l}{ }^{3} D_{1}=-0.0069 c_{P}+0.0100 s_{P} \\
{ }^{3} S_{1}=+0.0153 c_{P}+0.0111 s_{P}\end{array}$ & & 1601 \\
\hline & $D * B$ & & $\begin{array}{l}{ }^{3} S_{1}=+0.0153 c_{P}+0.0111 s_{P} \\
{ }^{3} D_{1}=-0.0053 c_{P}+0.007 s_{P}\end{array}$ & 1.67 & 16.01 \\
\hline & $D^{*} B^{*}$ & & ${ }^{3} S_{1}=-0.0121 s_{P}$ & 7.32 & 70.18 \\
\hline & & & $\begin{array}{l}{ }^{3} D_{1}=-0.0417 s_{P} \\
{ }^{5} D_{1}=-0.0503 c_{P}\end{array}$ & & \\
\hline & total & & & 10.43 & 100 \\
\hline \multirow[t]{8}{*}{$B_{c}\left(4^{3} P_{2}\right)$} & $D B$ & & ${ }^{1} D_{2}=-0.0013$ & 0.01 & 0.14 \\
\hline & $D B^{*}$ & & ${ }^{3} D_{2}=+0.0088$ & 0.52 & 7.38 \\
\hline & $D^{*} B$ & & ${ }^{3} D_{2}=+0.0001$ & 0.1 & 1.42 \\
\hline & $D^{*} B^{*}$ & & ${ }^{5} S_{2}=+0.0246$ & 6.41 & 91.01 \\
\hline & & & ${ }^{1} D_{2}=+0.0126$ & & \\
\hline & & & ${ }^{5} D_{2}=-0.0335$ & & \\
\hline & $D_{s} B_{s}$ & & ${ }^{1} D_{2}=-0.001$ & 0.003 & 0.004 \\
\hline & total & & & 7.043 & 100 \\
\hline
\end{tabular}

of $\sim 2 \%$ and (ii) $B_{c}^{ \pm} \rightarrow J / \psi l^{ \pm} \nu_{l} \rightarrow l^{\prime-} l^{\prime+} l^{ \pm} \nu_{l}$ having combined BR of $0.21 \%$ and detection efficiency of $\sim 4 \%$ (see Table XV for the branching ratios). We calculate the number of events of various decay chains of excited $B_{c}$ states below the $B D$ threshold at the LHC for integrated luminosity $L=100 \mathrm{fb}^{-1}$. The values reported in Tables XVI-XXII include the events observed through both of the golden channels. The decay chains having yield less than 100 are not included in these tables.

We include a factor of 2 to incorporate both charge conjugate states of $B_{c}$. It is noted that our mass calculations show that $3 S, 2 P, 2 D$, and $1 F$ states are below but close to the $B D$ threshold (energy gap $<0.15 \mathrm{GeV}$ ). These results significantly differ from the mass predictions given in Refs. [5,6,8]. Reference [5] shows that $3 S, 2^{3} P_{2}, 2 D$, and $1 F$ states are above the $B D$ threshold, whereas Ref. [8] shows that three multiplets of $2 P$ states are above the $B D$ threshold along with $3 S, 2 D$, and $1 F$ states. Reference [6] also shows that $3 S$ and three multiplets of $2 P$ states are above the $B D$ threshold. The result is that these states, which are expected to be observed through radiative and hadronic transition according to our mass predictions, are unlikely to be observed in these references. This also gives significantly different branching ratios and predicted strong decay widths. Thus the experimenters should treat our predictions of branching ratios and strong decay widths of the states close to the $B D$ threshold cautiously. Besides this caveat, there are no results available for the production cross sections of $3 S, 2 D$, and $1 F$ states in $p p$ collision; therefore, we abstain from making any predictions of event rates for these states.

These results show that in the LHC it is possible to produce a sufficient number of events corresponding to different decay chains of the excited $B_{c}$ states below the $B D$ threshold. The task of event reconstruction becomes much easier when an excited $B_{c}$ state directly decays to the ground state through $E 1 / M 1$ or hadronic transitions. Tables VII and VIII show that $1^{3} S_{1}, 2^{3} S_{1}, 1 P_{1}, 1 P_{1}^{\prime}, 2 P_{1}$, 
TABLE X. Partial widths and branching ratios for strong, radiative, and hadronic transitions for the $1 D$ states of $B_{c}$ mesons.

\begin{tabular}{|c|c|c|c|c|c|}
\hline Meson & Decay Mode & Photon Energy MeV & Amplitude & $\Gamma_{\text {thy }} \mathrm{MeV}$ & B.R \\
\hline \multirow{6}{*}{$\overline{B_{c}\left(1^{3} D_{1}\right)}$} & $B_{c}\left(1^{3} S_{1}\right)+\pi \pi$ & & & $0.042 \mathrm{keV}$ & 0.07 \\
\hline & $B_{c}\left(1^{3} P_{0}\right) \gamma$ & 206.78 & $\left\langle 1^{3} P_{0}|r| 1^{3} D_{1}\right\rangle=3.3708$ & $40.55 \mathrm{keV}$ & 63.97 \\
\hline & $B_{c}\left(1 P_{1}\right) \gamma$ & 188.33 & $\left\langle 1^{3} P_{1}|r| 1^{3} D_{1}\right\rangle=3.3708$ & $7.67 \mathrm{keV}$ & 12.1 \\
\hline & $B_{c}\left(1 P_{1}^{\prime}\right) \gamma$ & 182.50 & $\left\langle 1^{3} P_{1}|r| 1^{3} D_{1}\right\rangle=3.3708$ & $13.93 \mathrm{keV}$ & 21.98 \\
\hline & $B_{c}\left(1^{3} P_{2}\right) \gamma$ & 173.74 & $\left\langle 1^{3} P_{2}|r| 1^{3} D_{1}\right\rangle=3.3708$ & $1.2 \mathrm{keV}$ & 1.89 \\
\hline & total & & & $63.39 \mathrm{keV}$ & 100 \\
\hline \multirow{6}{*}{$B_{c}\left(1 D_{2}\right)$} & $B_{c}\left(1^{1} S_{0}\right)+\pi \pi$ & & & $0.035 \mathrm{keV}$ & 0.06 \\
\hline & $B_{c}\left(1^{3} S_{1}\right)+\pi \pi$ & & & $0.017 \mathrm{keV}$ & 0.03 \\
\hline & $B_{c}\left(1^{3} P_{2}\right) \gamma$ & 177.63 & $\left\langle 1^{3} P_{2}|r| 1^{3} D_{2}\right\rangle=3.3708$ & $4.62 \mathrm{keV}$ & 8.12 \\
\hline & $B_{c}\left(1 P_{1}\right) \gamma$ & 192.22 & $\begin{array}{l}\left\langle 1^{3} P_{1}|r| 1^{3} D_{2}\right\rangle=3.3708 \\
\left\langle 1^{1} P_{1}|r| 1^{1} D_{2}\right\rangle=3.3461\end{array}$ & $52.26 \mathrm{keV}$ & 91.8 \\
\hline & $B_{c}\left(1 P_{1}^{\prime}\right) \gamma$ & 186.39 & $\begin{array}{l}\left\langle 1^{3} P_{1}|r| 1^{3} D_{2}\right\rangle=3.3708 \\
\left\langle 1^{1} P_{1}|r| 1^{1} D_{2}\right\rangle=3.3461\end{array}$ & $2.6 \times 10^{-4} \mathrm{keV}$ & 0.0005 \\
\hline & total & & & $56.93 \mathrm{keV}$ & 100 \\
\hline \multirow[t]{6}{*}{$B_{c}\left(1 D_{2}^{\prime}\right)$} & $B_{c}\left(1^{1} S_{0}\right)+\pi \pi$ & & & $0.023 \mathrm{keV}$ & 0.04 \\
\hline & $B_{c}\left(1^{3} S_{1}\right)+\pi \pi$ & & & $0.026 \mathrm{keV}$ & 0.05 \\
\hline & $B_{c}\left(1^{3} P_{2}\right) \gamma$ & 177.63 & $\left\langle 1^{3} P_{2}|r| 1^{3} D_{2}\right\rangle=3.3708$ & $6.95 \mathrm{keV}$ & 13.32 \\
\hline & $B_{c}\left(1 P_{1}\right) \gamma$ & 192.22 & $\begin{array}{l}\left\langle 1^{3} P_{1}|r| 1^{3} D_{2}\right\rangle=3.3708 \\
\left\langle 1^{1} P_{1}|r| 1^{1} D_{2}\right\rangle=3.3461\end{array}$ & $0.9 \mathrm{keV}$ & 1.72 \\
\hline & $B_{c}\left(1 P_{1}^{\prime}\right) \gamma$ & 186.39 & $\begin{array}{l}\left\langle 1^{3} P_{1}|r| 1^{3} D_{2}\right\rangle=3.3708 \\
\left\langle 1^{1} P_{1}|r| 1^{1} D_{2}\right\rangle=3.3461\end{array}$ & $44.29 \mathrm{keV}$ & 84.86 \\
\hline & total & & & $52.19 \mathrm{keV}$ & 100 \\
\hline \multirow[t]{3}{*}{$B_{c}\left(1^{3} D_{3}\right)$} & $B_{c}\left(1^{3} S_{1}\right)+\pi \pi$ & & & $0.043 \mathrm{keV}$ & 0.09 \\
\hline & $B_{c}\left(1^{3} P_{2}\right) \gamma$ & 179.58 & $\left\langle 1^{3} P_{2}|r| 1^{3} D_{3}\right\rangle=3.3708$ & $47.81 \mathrm{keV}$ & 99.91 \\
\hline & total & & & $47.85 \mathrm{keV}$ & 100 \\
\hline
\end{tabular}

TABLE XI. Partial widths and branching ratios for strong, radiative, and hadronic transitions for the $2 D$ states of $B_{c}$ mesons.

\begin{tabular}{|c|c|c|c|c|c|}
\hline Meson & Decay mode & Photon energy $\mathrm{MeV}$ & Amplitude & $\Gamma_{\text {thy }} \mathrm{MeV}$ & B.R $(\%)$ \\
\hline \multirow[t]{10}{*}{$B_{c}\left(2^{3} D_{1}\right)$} & $B_{c}\left(2^{3} P_{0}\right) \gamma$ & 163.08 & $\left\langle 2^{3} P_{0}|r| 2^{3} D_{1}\right\rangle=4.4775$ & $35.09 \mathrm{keV}$ & 52.98 \\
\hline & $B_{c}\left(2 P_{1}\right) \gamma$ & 148.41 & $\left\langle 2^{3} P_{1}|r| 2^{3} D_{1}\right\rangle=4.4775$ & $6.62 \mathrm{keV}$ & 10.0 \\
\hline & $B_{c}\left(2 P_{1}^{\prime}\right) \gamma$ & 139.60 & $\left\langle 2^{3} P_{1}|r| 2^{3} D_{1}\right\rangle=4.4775$ & $11.0 \mathrm{keV}$ & 16.61 \\
\hline & $B_{c}\left(2^{3} P_{2}\right) \gamma$ & 132.73 & $\left\langle 2^{3} P_{2}|r| 2^{3} D_{1}\right\rangle=4.4775$ & $0.95 \mathrm{keV}$ & 1.43 \\
\hline & $B_{c}\left(1^{3} P_{0}\right) \gamma$ & 434.76 & $\left\langle 1^{3} P_{0}|r| 2^{3} D_{1}\right\rangle=0.3469$ & $3.99 \mathrm{keV}$ & 6.02 \\
\hline & $B_{c}\left(1 P_{1}\right) \gamma$ & 416.94 & $\left\langle 1^{3} P_{1}|r| 2^{3} D_{1}\right\rangle=0.3469$ & $0.88 \mathrm{keV}$ & 1.33 \\
\hline & $B_{c}\left(1 P_{1}^{\prime}\right) \gamma$ & 411.30 & $\left\langle 1^{3} P_{1}|r| 2^{3} D_{1}\right\rangle=0.3469$ & $1.69 \mathrm{keV}$ & 2.55 \\
\hline & $B_{c}\left(1^{3} P_{2}\right) \gamma$ & 402.84 & $\left\langle 1^{3} P_{2}|r| 2^{3} D_{1}\right\rangle=0.3469$ & $0.16 \mathrm{keV}$ & 0.24 \\
\hline & $B_{c}\left(1^{3} F_{2}\right) \gamma$ & 82.32 & $\left\langle 1^{3} F_{2}|r| 2^{3} D_{1}\right\rangle=-3.1023$ & $5.85 \mathrm{keV}$ & 8.83 \\
\hline & total & & & $66.23 \mathrm{keV}$ & 100 \\
\hline
\end{tabular}


TABLE XI. (Continued)

\begin{tabular}{|c|c|c|c|c|c|}
\hline Meson & Decay mode & Photon energy $\mathrm{MeV}$ & Amplitude & $\Gamma_{\text {thy }} \mathrm{MeV}$ & B.R $(\%)$ \\
\hline \multirow[t]{10}{*}{$B_{c}\left(2 D_{2}\right)$} & $B_{c}\left(2^{3} P_{2}\right) \gamma$ & 136.66 & $\left\langle 2^{3} P_{2}|r| 2^{3} D_{2}\right\rangle=4.4775$ & $3.71 \mathrm{keV}$ & 5.82 \\
\hline & $B_{c}\left(2 P_{1}\right) \gamma$ & 152.33 & $\left\langle 2^{3} P_{1}|r| 2^{3} D_{2}\right\rangle=4.4775$ & $45.81 \mathrm{keV}$ & 71.84 \\
\hline & $B_{c}\left(2 P_{1}^{\prime}\right) \gamma$ & 143.52 & $\begin{array}{l}\left\langle 2^{1} P_{1}|r| 2^{1} D_{2}\right\rangle=4.4392 \\
\left\langle 2^{3} P_{1}|r| 2^{3} D_{2}\right\rangle=4.4775 \\
\left\langle 2^{1} P_{1}|r| 2^{1} D_{2}\right\rangle=4.4392\end{array}$ & $3.2 \times 10^{-4} \mathrm{keV}$ & 0.001 \\
\hline & $B_{c}\left(1^{3} P_{2}\right) \gamma$ & 406.61 & $\left\langle 1^{3} P_{2}|r| 2^{3} D_{2}\right\rangle=0.3469$ & $0.59 \mathrm{keV}$ & 0.93 \\
\hline & $B_{c}\left(1 P_{1}\right) \gamma$ & 420.71 & $\begin{array}{l}\left\langle 1^{3} P_{1}|r| 2^{3} D_{2}\right\rangle=0.3469 \\
\left\langle 1^{1} P_{1}|r| 2^{1} D_{2}\right\rangle=0.3603\end{array}$ & $6.17 \mathrm{keV}$ & 9.68 \\
\hline & $B_{c}\left(1 P_{1}^{\prime}\right) \gamma$ & 415.07 & $\begin{array}{l}\left\langle 1^{3} P_{1}|r| 2^{3} D_{2}\right\rangle=0.3469 \\
\left\langle 1^{1} P_{1}|r| 2^{1} D_{2}\right\rangle=0.3603\end{array}$ & $2.1 \times 10^{-3} \mathrm{keV}$ & 0.003 \\
\hline & $B_{c}\left(1^{3} F_{2}\right) \gamma$ & 86.27 & $\left\langle 1^{3} F_{2}|r| 2^{3} D_{2}\right\rangle=-3.1023$ & $0.3 \mathrm{keV}$ & 0.47 \\
\hline & $B_{c}\left(1 F_{3}\right) \gamma$ & 89.43 & $\begin{aligned}\left\langle 1^{3} F_{3}|r| 2^{3} D_{2}\right\rangle & =-3.1023, \\
\left\langle 1^{1} F_{3}|r| 2^{1} D_{2}\right\rangle & =-3.1085\end{aligned}$ & $7.16 \mathrm{keV}$ & 11.23 \\
\hline & $B_{c}\left(1 F_{3}^{\prime}\right) \gamma$ & 82.51 & $\begin{aligned}\left\langle 1^{3} F_{3}|r| 2^{3} D_{2}\right\rangle & =-3.1023 \\
\left\langle 1^{1} F_{3}|r| 2^{1} D_{2}\right\rangle & =-3.1085\end{aligned}$ & $0.02 \mathrm{keV}$ & 0.03 \\
\hline & total & & & $63.76 \mathrm{keV}$ & 100 \\
\hline \multirow[t]{10}{*}{$B_{c}\left(2 D_{2}^{\prime}\right)$} & $B_{c}\left(2^{3} P_{2}\right) \gamma$ & 136.66 & $\left\langle 2^{3} P_{2}|r| 2^{3} D_{2}\right\rangle=4.4775$ & $5.58 \mathrm{keV}$ & 10.26 \\
\hline & $B_{c}\left(2 P_{1}\right) \gamma$ & 152.33 & $\begin{aligned}\left\langle 2^{3} P_{1}|r| 2^{3} D_{2}\right\rangle & =4.4775 \\
\left\langle 2^{1} P_{1}|r| 2^{1} D_{2}\right\rangle & =4.4392\end{aligned}$ & $0.79 \mathrm{keV}$ & 1.45 \\
\hline & $B_{c}\left(2 P_{1}^{\prime}\right) \gamma$ & 143.52 & $\begin{aligned}\left\langle 2^{3} P_{1}|r| 2^{3} D_{2}\right\rangle & =4.4775 \\
\left\langle 2^{1} P_{1}|r| 2^{1} D_{2}\right\rangle & =4.4392\end{aligned}$ & $35.64 \mathrm{keV}$ & 65.56 \\
\hline & $B_{c}\left(1^{3} P_{2}\right) \gamma$ & 406.61 & $\left\langle 1^{3} P_{2}|r| 2^{3} D_{2}\right\rangle=0.3469$ & $0.88 \mathrm{keV}$ & 1.62 \\
\hline & $B_{c}\left(1 P_{1}\right) \gamma$ & 420.71 & $\begin{array}{l}\left\langle 1^{3} P_{1}|r| 2^{3} D_{2}\right\rangle=0.3469 \\
\left\langle 1^{1} P_{1}|r| 2^{1} D_{2}\right\rangle=0.3603\end{array}$ & $0.14 \mathrm{keV}$ & 0.26 \\
\hline & $B_{c}\left(1 P_{1}^{\prime}\right) \gamma$ & 415.07 & $\begin{array}{l}\left\langle 1^{3} P_{1}|r| 2^{3} D_{2}\right\rangle=0.3469 \\
\left\langle 1^{1} P_{1}|r| 2^{1} D_{2}\right\rangle=0.3603\end{array}$ & $5.37 \mathrm{keV}$ & 9.88 \\
\hline & $B_{c}\left(1^{3} F_{2}\right) \gamma$ & 86.27 & $\left\langle 1^{3} F_{2}|r| 2^{3} D_{2}\right\rangle=-3.1023$ & $0.45 \mathrm{keV}$ & 0.83 \\
\hline & $B_{c}\left(1 F_{3}\right) \gamma$ & 89.43 & $\begin{array}{l}\left\langle 1^{3} F_{2}|r| 2^{3} D_{2}\right\rangle=-3.1023 \\
\left\langle 1^{1} F_{2}|r| 2^{1} D_{2}\right\rangle=-3.1085\end{array}$ & $1.7 \times 10^{-6} \mathrm{keV}$ & $\sim 0$ \\
\hline & $B_{c}\left(1 F_{3}^{\prime}\right) \gamma$ & 82.51 & $\begin{array}{l}\left\langle 1^{3} F_{2}|r| 2^{3} D_{2}\right\rangle=-3.1023 \\
\left\langle 1^{1} F_{2}|r| 2^{1} D_{2}\right\rangle=-3.1085\end{array}$ & $5.51 \mathrm{keV}$ & 10.14 \\
\hline & total & & & $54.36 \mathrm{keV}$ & 100 \\
\hline \multirow[t]{7}{*}{$B_{c}\left(2^{3} D_{3}\right)$} & $B_{c}\left(2^{3} P_{2}\right) \gamma$ & 139.60 & $\left\langle 2^{3} P_{2}|r| 2^{3} D_{3}\right\rangle=4.4775$ & $39.62 \mathrm{keV}$ & 74.5 \\
\hline & $B_{c}\left(1^{3} P_{2}\right) \gamma$ & 409.44 & $\left\langle 1^{3} P_{2}|r| 2^{3} D_{3}\right\rangle=0.3469$ & $6.0 \mathrm{keV}$ & 11.28 \\
\hline & $B_{c}\left(1^{3} F_{4}\right) \gamma$ & 89.72 & $\left\langle 1^{3} F_{4}|r| 2^{3} D_{3}\right\rangle=-3.1023$ & $6.96 \mathrm{keV}$ & 13.09 \\
\hline & $B_{c}\left(1^{3} F_{2}\right) \gamma$ & 89.23 & $\left\langle 1^{3} F_{2}|r| 2^{3} D_{3}\right\rangle=-3.1023$ & $0.02 \mathrm{keV}$ & 0.04 \\
\hline & $B_{c}\left(1 F_{3}\right) \gamma$ & 92.39 & $\left\langle 1^{3} F_{3}|r| 2^{3} D_{3}\right\rangle=-3.1023$ & $0.28 \mathrm{keV}$ & 0.53 \\
\hline & $B_{c}\left(1 F_{3}^{\prime}\right) \gamma$ & 85.48 & $\left\langle 1^{3} F_{3}|r| 2^{3} D_{3}\right\rangle=-3.1023$ & $0.3 \mathrm{keV}$ & 0.56 \\
\hline & total & & & $53.18 \mathrm{keV}$ & 100 \\
\hline
\end{tabular}

$2 P_{1}^{\prime}$ states can directly decay to $B_{c}$ ground states through $E 1 / M 1$ transitions. All these direct transitions also appear in Tables XVI, XVII, XIX, and XX of decay chains as their yield is much higher than 100 . The case of $2{ }^{3} S_{1} \rightarrow 1^{1} S_{0}+\gamma$ is particulary interesting. Only 2650 events are expected in this case owing to the small value of its $\mathrm{BR}(\approx 2.75 \%)$ despite having relatively large production cross section of $2^{3} S_{1}$ state. Therefore, the best way to search this state is via $2^{3} S_{1} \stackrel{\gamma}{\rightarrow} 1^{3} P_{2} \stackrel{\gamma}{\rightarrow} 1^{3} S_{1} \stackrel{\gamma}{\rightarrow} 1^{1} S_{0}$ or $2^{3} S_{1} \stackrel{\pi \pi}{\rightarrow} 1^{3} S_{1} \stackrel{\gamma}{\rightarrow} 1^{1} S_{0}$ for which the expected numbers of events are $2.78 \times 10^{4}$ and $2.58 \times$ $10^{4}$ respectively as shown in the Table XVI. Tables VII and $\mathrm{X}$ show that $2^{1} S_{0}, 1 D_{2}$, and $1 D_{2}^{\prime}$ states can directly decay to 
TABLE XII. Partial widths and branching ratios for strong, radiative, and hadronic transitions for the $3 D$ states of $B_{c}$ mesons.

\begin{tabular}{|c|c|c|c|c|c|}
\hline Meson & Decay mode & Photon energy $\mathrm{MeV}$ & Amplitude & $\Gamma_{\text {thy }} \mathrm{MeV}$ & B.R $(\%)$ \\
\hline \multirow{4}{*}{$B_{c}\left(3^{3} D_{1}\right)$} & $D B$ & & ${ }^{1} P_{1}=-0.0126$ & 0.91 & 59.48 \\
\hline & $D B^{*}$ & & ${ }^{3} P_{1}=+0.0108$ & 0.55 & 35.95 \\
\hline & $D^{*} B$ & & ${ }^{3} P_{1}=+0.0122$ & 0.07 & 4.58 \\
\hline & total & & & 1.53 & 100 \\
\hline \multirow[t]{3}{*}{$B_{c}\left(3 D_{2}\right)$} & $D B^{*}$ & & $\begin{array}{l}{ }^{3} P_{2}=-0.0127 c_{D}+0.0156 s_{D} \\
{ }^{3} F_{2}=-0.0151\end{array}$ & 1.81 & 99.81 \\
\hline & $D^{*} B$ & & $\begin{array}{l}{ }^{3} F_{2}=-0.0151 c_{D}-0.0123 s_{D} \\
{ }^{3} P_{2}=-0.0281 c_{D}+0.0344 s_{D} \\
{ }^{3} F_{2}=-0.0014 c_{D}-0.0011 s_{D}\end{array}$ & $3.5 \times 10^{-3}$ & 0.19 \\
\hline & total & & & 1.81 & 100 \\
\hline \multirow[t]{3}{*}{$B_{c}\left(3 D_{2}^{\prime}\right)$} & $D B^{*}$ & & $\begin{array}{l}{ }^{3} D_{2}=+0.0156 c_{D}+0.0127 s_{D} \\
{ }^{3} F_{2}=-0.0123 c_{D}+0.0151 s_{D}\end{array}$ & 1.93 & 47.3 \\
\hline & $D^{*} B$ & & $\begin{array}{l}{ }^{3} D_{2}=+0.0344 c_{D}+0.0281 s_{D} \\
{ }^{3} F_{2}=-0.0011 c_{D}+0.0014 s_{D}\end{array}$ & 2.15 & 52.7 \\
\hline & total & & & 4.08 & 100 \\
\hline \multirow[t]{4}{*}{$B_{c}\left(3^{3} D_{3}\right)$} & $D B$ & & ${ }^{1} F_{3}=+0.0002$ & 0.0002 & 0.02 \\
\hline & $D B^{*}$ & & ${ }^{3} F_{3}=-0.0143$ & 0.99 & 98.98 \\
\hline & $D^{*} B$ & & ${ }^{3} F_{3}=-0.0027$ & 0.01 & 1.0 \\
\hline & total & & & 1.0 & 100 \\
\hline
\end{tabular}

TABLE XIII. Partial widths and branching ratios for strong, radiative, and hadronic transitions for the $4 D$ states of $B_{c}$ mesons.

\begin{tabular}{|c|c|c|c|c|c|}
\hline Meson & Decay mode & Photon energy MeV & Amplitude $(\mathcal{M})$ & $\Gamma_{\text {thy }} \mathrm{MeV}$ & B.R $(\%)$ \\
\hline \multirow[t]{8}{*}{$B_{c}\left(4^{3} D_{1}\right)$} & $D B$ & & ${ }^{1} P_{1}=-0.0178$ & 2.95 & 72.25 \\
\hline & $D B^{*}$ & & ${ }^{3} P_{1}=+0.0023$ & 0.05 & 1.22 \\
\hline & $D^{*} B$ & & ${ }^{3} P_{1}=+0.0061$ & 0.27 & 6.61 \\
\hline & $D^{*} B^{*}$ & & ${ }^{1} P_{1}=+0.0009$ & 0.81 & 19.84 \\
\hline & & & $\begin{array}{l}{ }^{5} P_{1}=-0.0004 \\
{ }^{5} F_{1}=-0.0114\end{array}$ & & \\
\hline & $D_{s} B_{s}$ & & ${ }^{1} P_{1}=-0.0007$ & 0.003 & 0.07 \\
\hline & $D_{s} B_{s}^{*}$ & & ${ }^{3} P_{1}=+0.0002$ & $1.5 \times 10^{-4}$ & $\sim 0$ \\
\hline & total & & & 4.08 & 100 \\
\hline \multirow[t]{7}{*}{$B_{c}\left(4 D_{2}\right)$} & $D B^{*}$ & & ${ }^{3} D_{2}=-0.0028 c_{D}+0.0040 s_{D}$ & 0.61 & 14.94 \\
\hline & & & ${ }^{3} F_{2}=0.0063 c_{D}+0.0055 s_{D}$ & & \\
\hline & $D^{*} B$ & & ${ }^{3} D_{2}=-0.0072 c_{D}+0.0089 s_{D}$ & 1.03 & 25.23 \\
\hline & $D^{*} B^{*}$ & & $\begin{array}{c}{ }^{3} F_{2}=0.0092 c_{D}+0.0074 s_{D} \\
{ }^{3} P_{2}=-0.0005 c_{D},{ }^{3} F_{2}=-0.0093 c_{D} \\
{ }^{5} P_{2}=+0.0001 s_{D},{ }^{5} F_{2}=+0.0109 s_{D}\end{array}$ & 0.65 & 15.92 \\
\hline & $D_{s} B_{s}^{*}$ & & ${ }^{3} D_{2}=-0.0001 c_{D}+0.0001 s_{D}$ & $7.0 \times 10^{-4}$ & 0.02 \\
\hline & $D_{s}^{*} B_{s}$ & & $\begin{array}{l}{ }^{3} F_{2}=+0.0003 c_{D}+0.0003 s_{D} \\
{ }^{3} D_{2}=-0.0021 c_{D}+0.0025 s_{D} \\
{ }^{3} F_{2}=+0.0001 c_{D}+0.0001 s_{D}\end{array}$ & $3.7 \times 10^{-5}$ & $\sim 0$ \\
\hline & total & & & 2.29 & 100 \\
\hline
\end{tabular}


TABLE XIII. (Continued)

\begin{tabular}{|c|c|c|c|c|c|}
\hline Meson & Decay mode & Photon energy $\mathrm{MeV}$ & Amplitude $(\mathcal{M})$ & $\Gamma_{\text {thy }} \mathrm{MeV}$ & B.R $(\%)$ \\
\hline \multirow[t]{6}{*}{$B_{c}\left(4 D_{2}^{\prime}\right)$} & $D B^{*}$ & & ${ }^{3} D_{2}=0.0040 c_{D}+0.0028 s_{D}$ & 0.21 & 11.28 \\
\hline & $D^{*} B$ & & $\begin{aligned}{ }^{3} F_{2} & =0.0055 c_{D}-0.0063 s_{D} \\
{ }^{3} D_{2} & =0.0089 c_{D}+0.0072 s_{D}\end{aligned}$ & 0.96 & 51.58 \\
\hline & $D^{*} B^{*}$ & & $\begin{array}{c}{ }^{3} F_{2}=0.0074 c_{D}-0.0092 s_{D} \\
{ }^{3} P_{2}=+0.0005 s_{D},{ }^{3} F_{2}=+0.0093 s_{D}\end{array}$ & 0.68 & 36.54 \\
\hline & $D_{s} B_{s}^{*}$ & & $\begin{array}{c}{ }^{5} P_{2}=+0.0001 c_{D},{ }^{5} F_{2}=+0.0109 c_{D} \\
{ }^{3} D_{2}=+0.0001 c_{D}+0.0001 s_{D} \\
{ }^{3} F_{2}=+0.0003 c_{D}-0.0003 s_{D}\end{array}$ & $1.5 \times 10^{-4} 0.01$ & \\
\hline & $D_{s}^{*} B_{s}$ & & $\begin{array}{l}{ }^{3} D_{2}=+0.0025 c_{D}+0.0021 s_{D} \\
{ }^{3} F_{2}=+0.0001 c_{D}-0.0001 s_{D}\end{array}$ & 0.011 & 0.59 \\
\hline & total & & & 1.86 & 100 \\
\hline \multirow[t]{9}{*}{$B_{c}\left(4^{3} D_{3}\right)$} & $D B$ & & ${ }^{1} F_{3}=+0.0117$ & 1.3 & 44.19 \\
\hline & $D B^{*}$ & & ${ }^{3} F_{3}=-0.0074$ & 0.48 & 16.32 \\
\hline & $D^{*} B$ & & ${ }^{3} F_{3}=-0.0088$ & 0.58 & 19.72 \\
\hline & $D^{*} B^{*}$ & & ${ }^{5} P_{3}=-0.0024$ & 0.58 & 19.72 \\
\hline & & & $\begin{array}{l}{ }^{1} F_{3}=+0.0038 \\
{ }^{5} F_{3}=-0.0083\end{array}$ & & \\
\hline & $D_{s} B_{s}$ & & ${ }^{1} F_{3}=+0.0003$ & $4.4 \times 10^{-4}$ & 0.01 \\
\hline & $D_{s} B_{s}^{*}$ & & ${ }^{3} F_{3}=-0.0004$ & $8.9 \times 10^{-4}$ & 0.03 \\
\hline & $D_{s}^{*} B_{s}$ & & ${ }^{3} F_{3}=-0.0005$ & $3.5 \times 10^{-4}$ & 0.01 \\
\hline & total & & & 2.94 & 100 \\
\hline
\end{tabular}

TABLE XIV. Partial widths and branching ratios for strong, radiative, and hadronic transitions for the $1 F$ and $2 F$ states of $B_{c}$ mesons.

\begin{tabular}{|c|c|c|c|c|c|}
\hline Meson & Decay mode & Photon energy $\mathrm{MeV}$ & Amplitude $(\mathcal{M})$ & $\Gamma_{\text {thy }} \mathrm{MeV}$ & B.R $(\%)$ \\
\hline \multirow[t]{9}{*}{$B_{c}\left(1^{3} F_{2}\right)$} & $B_{c}\left(1^{3} P_{0}\right)+\pi \pi$ & & & $0.0004 \mathrm{keV}$ & 0.0007 \\
\hline & $B_{c}\left(1^{3} P_{2}\right)+\pi \pi$ & & & $0.00004 \mathrm{keV}$ & 0.0001 \\
\hline & $B_{c}\left(1 P_{1}\right)+\pi \pi$ & & & $0.0001 \mathrm{keV}$ & 0.0002 \\
\hline & $B_{c}\left(1 P_{1}^{\prime}\right)+\pi \pi$ & & & $0.0002 \mathrm{keV}$ & 0.0004 \\
\hline & $B_{c}\left(1^{3} D_{1}\right) \gamma$ & 154.46 & $\left\langle 1^{3} D_{1}|r| 1^{3} F_{2}\right\rangle=4.4823$ & $48.41 \mathrm{keV}$ & 85.02 \\
\hline & $B_{c}\left(1 D_{2}\right) \gamma$ & 150.54 & $\left\langle 1^{3} D_{2}|r| 1^{3} F_{2}\right\rangle=4.4823$ & $3.32 \mathrm{keV}$ & 5.83 \\
\hline & $B_{c}\left(1 D_{2}^{\prime}\right) \gamma$ & 150.54 & $\left\langle 1^{3} D_{2}|r| 1^{3} F_{2}\right\rangle=4.4823$ & $4.98 \mathrm{keV}$ & 8.75 \\
\hline & $B_{c}\left(1^{3} D_{3}\right) \gamma$ & 148.59 & $\left\langle 1^{3} D_{3}|r| 1^{3} F_{2}\right\rangle=4.4823$ & $0.23 \mathrm{keV}$ & 0.4 \\
\hline & total & & & $56.94 \mathrm{keV}$ & 100 \\
\hline \multirow[t]{6}{*}{$B_{c}\left(1 F_{3}\right)$} & $B_{c}\left(1^{3} P_{2}\right)+\pi \pi$ & & & $0.0001 \mathrm{keV}$ & 0.0002 \\
\hline & $B_{c}\left(1 P_{1}\right)+\pi \pi$ & & & $0.001 \mathrm{keV}$ & 0.002 \\
\hline & $B_{c}\left(1^{3} D_{3}\right) \gamma$ & 145.46 & $\left\langle 1^{3} D_{3}|r| 1^{3} F_{3}\right\rangle=4.4823$ & $2.29 \mathrm{keV}$ & 4.59 \\
\hline & $B_{c}\left(1 D_{2}\right) \gamma$ & 147.41 & $\begin{array}{l}\left\langle 1^{3} D_{2}|r| 1^{3} F_{3}\right\rangle=4.4823 \\
\left\langle 1^{1} D_{2}|r| 1^{1} F_{3}\right\rangle=4.4769\end{array}$ & $47.64 \mathrm{keV}$ & 95.41 \\
\hline & $B_{c}\left(1 D_{2}^{\prime}\right) \gamma$ & 147.41 & $\begin{aligned}\left\langle 1^{3} D_{2}|r| 1^{3} F_{3}\right\rangle & =4.4823 \\
\left\langle 1^{1} D_{2}|r| 1^{1} F_{3}\right\rangle & =4.4769\end{aligned}$ & $0.0001 \mathrm{keV}$ & 0.0002 \\
\hline & total & & & $49.93 \mathrm{keV}$ & 100 \\
\hline
\end{tabular}


TABLE XIV. (Continued)

\begin{tabular}{|c|c|c|c|c|c|}
\hline Meson & Decay mode & Photon energy MeV & Amplitude $(\mathcal{M})$ & $\Gamma_{\text {thy }} \mathrm{MeV}$ & B.R $(\%)$ \\
\hline \multirow[t]{7}{*}{$\overline{B_{c}\left(1 F_{3}^{\prime}\right)}$} & $B_{c}\left(1^{3} P_{2}\right)+\pi \pi$ & & & $0.0001 \mathrm{keV}$ & 0.0002 \\
\hline & $B_{c}\left(1 P_{1}\right)+\pi \pi$ & & & $2 \times 10^{-5} \mathrm{keV}$ & $\sim 0$ \\
\hline & $B_{c}\left(1 P_{1}^{\prime}\right)+\pi \pi$ & & & $0.001 \mathrm{keV}$ & 0.002 \\
\hline & $B_{c}\left(1^{3} D_{3}\right) \gamma$ & 152.31 & $\left\langle 1^{3} D_{3}|r| 1^{3} F_{3}\right\rangle=4.4823$ & $3.51 \mathrm{keV}$ & 6.13 \\
\hline & $B_{c}\left(1 D_{2}\right) \gamma$ & 154.26 & $\begin{aligned}\left\langle 1^{3} D_{2}|r| 1^{3} F_{3}\right\rangle & =4.4823, \\
\left\langle 1^{1} D_{2}|r| 1^{1} F_{3}\right\rangle & =4.4769\end{aligned}$ & $0.18 \mathrm{keV}$ & 0.31 \\
\hline & $B_{c}\left(1 D_{2}^{\prime}\right) \gamma$ & 154.26 & $\begin{array}{l}\left\langle 1^{3} D_{2}|r| 1^{3} F_{3}\right\rangle=4.4823 \\
\left\langle 1^{1} D_{2}|r| 1^{1} F_{3}\right\rangle=4.4769\end{array}$ & $53.53 \mathrm{keV}$ & 93.55 \\
\hline & total & & & $57.22 \mathrm{keV}$ & 100 \\
\hline \multirow[t]{3}{*}{$B_{c}\left(1^{3} F_{4}\right)$} & $B_{c}\left(1^{3} P_{2}\right)+\pi \pi$ & & & $0.0005 \mathrm{keV}$ & 0.001 \\
\hline & $B_{c}\left(1^{3} D_{3}\right) \gamma$ & 148.10 & $\left\langle 1^{3} D_{3}|r| 1^{3} F_{4}\right\rangle=4.4823$ & $50.8 \mathrm{keV}$ & $\sim 100$ \\
\hline & total & & & $50.8 \mathrm{keV}$ & 100 \\
\hline \multirow[t]{3}{*}{$B_{c}\left(2^{3} F_{2}\right)$} & $D B$ & & ${ }^{1} D_{2}=+0.0277$ & 2.89 & 76.66 \\
\hline & $D B^{*}$ & & ${ }^{3} D_{2}=+0.0206$ & 0.88 & 23.34 \\
\hline & total & & & 3.77 & 100 \\
\hline \multirow[t]{2}{*}{$B_{c}\left(2 F_{3}\right)$} & $D B^{*}$ & & ${ }^{3} D_{3}=-0.0205 c_{F}+0.0237 s_{F}$ & 0.01 & 100 \\
\hline & & & ${ }^{3} G_{3}=-0.0017 c_{F}-0.0012 s_{F}$ & & \\
\hline \multirow[t]{2}{*}{$B_{c}\left(2 F_{3}^{\prime}\right)$} & $D B^{*}$ & & ${ }^{3} D_{3}=+0.0262 c_{F}+0.0227 s_{F}$ & 2.61 & 100 \\
\hline & & & ${ }^{3} G_{3}=-0.0019 c_{F}+0.0022 s_{F}$ & & \\
\hline \multirow[t]{3}{*}{$B_{c}\left(2^{3} F_{4}\right)$} & $D B$ & & ${ }^{1} G_{4}=+0.0099$ & 0.37 & 97.37 \\
\hline & $D B^{*}$ & & ${ }^{3} G_{4}=-0.002$ & 0.01 & 2.63 \\
\hline & total & & & 0.38 & 100 \\
\hline
\end{tabular}

TABLE XV. Branching ratios for the two golden channels of $B_{c}$ along the branching ratio of $J / \psi \rightarrow l^{+} l^{-}$.

\begin{tabular}{lc}
\hline \hline Decay process & Branching ratio (\%) \\
\hline$B_{c}^{ \pm} \rightarrow J / \psi \pi^{ \pm}$ & $0.111_{-0.010}^{+0.009}[46]$ \\
$B_{c}^{ \pm} \rightarrow J / \psi l^{ \pm} \nu_{l}$ & $1.73 \pm 0.05[46]$ \\
$J / \psi \rightarrow l^{+} l^{-}$ & $11.9 \pm 0.06[16]$ \\
\hline \hline
\end{tabular}

$B_{c}$ ground state through hadronic transitions. However, due to small BRs of hardonic transitions of $1 D_{2}$ and $1 D_{2}^{\prime}$ (less than $1 \%$ ), the resultant number of events are less than 100 and are not included in Table XXII. The best way to detect $D$ states is via double or triple photon emission as given in Table XXII. Our results given in Tables XVI-XXII of decay chains can help experimentalists in adopting the best strategies to discover and study properties of the excited $B_{c}$ states below the $B D$ threshold.

\section{CONCLUDING REMARKS}

In this paper we studied the properties of charmedbottom mesons including masses, radiative transitions, hadronic transitions, and the Okubo-Zweig-Iizuka (OZI) allowed strong decays. We have computed the spectrum of $B_{c}$ mesons up to $2 F$ states with a nonrelativistic quark
TABLE XVI. Decay chains of $1 S$ and $2 S$ states and expected number of events at the LHC.

\begin{tabular}{|c|c|c|}
\hline Initial state & Decay chain & Number of events \\
\hline $1^{3} S_{1}$ & $\underset{100 \%}{\stackrel{\gamma}{\longrightarrow}} B_{c}$ & $1.6 \times 10^{5}$ \\
\hline \multirow[t]{5}{*}{$2^{1} S_{0}$} & $\underset{51.42 \%}{\stackrel{\pi \pi}{\longrightarrow}} B_{c}$ & $2.96 \times 10^{4}$ \\
\hline & $\underset{34.23}{\stackrel{\gamma}{\longrightarrow}} 1 P_{1} \underset{68.78 \%}{\stackrel{\gamma}{\longrightarrow}} B_{c}$ & $1.35 \times 10^{4}$ \\
\hline & $\underset{34.23 \%}{\stackrel{\gamma}{\longrightarrow}} 1 P_{1} \underset{31.22}{\stackrel{\gamma}{\longrightarrow}} 1^{3} S_{1} \underset{100 \%}{\stackrel{\gamma}{\longrightarrow}} B_{c}$ & $6.15 \times 10^{3}$ \\
\hline & $\underset{14.01 \%}{\stackrel{\gamma}{\rightarrow}} 1 P_{1}^{\prime} \underset{35.56 \%}{\stackrel{\gamma}{\longrightarrow}} B_{c}$ & $2.86 \times 10^{3}$ \\
\hline & $\underset{14.01 \%}{\stackrel{\gamma}{\longrightarrow}} 1 P_{1}^{\prime} \underset{64.44 \%}{\stackrel{\gamma}{\longrightarrow}} 1^{3} S_{1} \underset{100 \%}{\stackrel{\gamma}{\longrightarrow}} B_{c}$ & $5.19 \times 10^{3}$ \\
\hline \multirow[t]{8}{*}{$2^{3} S_{1}$} & $\underset{26.76 \%}{\stackrel{\pi \pi}{\longrightarrow}} 1^{3} S_{1} \underset{100 \%}{\stackrel{\gamma}{\longrightarrow}} B_{c}$ & $2.58 \times 10^{4}$ \\
\hline & $\underset{28.91 \%}{\stackrel{\gamma}{\longrightarrow}} 1^{3} P_{2} \underset{100 \%}{\stackrel{\gamma}{\rightarrow}} 1^{3} S_{1} \underset{100 \%}{\stackrel{\gamma}{\longrightarrow}} B_{c}$ & $2.78 \times 10^{4}$ \\
\hline & $\underset{2.75 \%}{\stackrel{\gamma}{\longrightarrow}} B_{c}$ & $2.65 \times 10^{3}$ \\
\hline & $\underset{9.59 \%}{\stackrel{\gamma}{\longrightarrow}} 1 P_{1} \underset{68.78 \%}{\stackrel{\gamma}{\longrightarrow}} B_{c}$ & $6.35 \times 10^{3}$ \\
\hline & $\underset{9.59 \%}{\stackrel{\gamma}{\longrightarrow}} 1 P_{1} \underset{31.22 \%}{\stackrel{\gamma}{\longrightarrow}} 1^{3} S_{1} \underset{100 \%}{\stackrel{\gamma}{\longrightarrow}} B_{c}$ & $2.88 \times 10^{3}$ \\
\hline & $\underset{15.76 \%}{\stackrel{\gamma}{\rightarrow}} 1 P_{1}^{\prime} \underset{35.56 \%}{\stackrel{\gamma}{\longrightarrow}} B_{c}$ & $5.4 \times 10^{3}$ \\
\hline & $\underset{15.76 \%}{\stackrel{\gamma}{\longrightarrow}} 1 P_{1}^{\prime} \underset{64.44}{\stackrel{\gamma}{\longrightarrow}} 1^{3} S_{1} \underset{100 \%}{\stackrel{\gamma}{\longrightarrow}} B_{c}$ & $9.78 \times 10^{3}$ \\
\hline & $\underset{16.23 \%}{\stackrel{\gamma}{\longrightarrow}} 1^{3} P_{0} \underset{100 \%}{\stackrel{\gamma}{\longrightarrow}} 1^{3} S_{1} \underset{100 \%}{\stackrel{\gamma}{\longrightarrow}} B_{c}$ & $1.56 \times 10^{4}$ \\
\hline
\end{tabular}


TABLE XVII. Decay chains of $1 P$ states and expected number of events at the LHC.

\begin{tabular}{|c|c|c|}
\hline Initial state & Decay chain & Number of events \\
\hline $1^{3} P_{2}$ & $\underset{100 \%}{\stackrel{\gamma}{\longrightarrow}} 1^{3} S_{1} \underset{100 \%}{\stackrel{\gamma}{\longrightarrow}} B_{c}$ & $2.6 \times 10^{4}$ \\
\hline \multirow[t]{2}{*}{$1 P_{1}^{\prime}$} & $\underset{35.56 \%}{\stackrel{\gamma}{\longrightarrow}} B_{c}$ & $6.53 \times 10^{3}$ \\
\hline & $\underset{64.44 \%}{\stackrel{\gamma}{\longrightarrow}} 1^{3} S_{1} \underset{100 \%}{\stackrel{\gamma}{\longrightarrow}} B_{c}$ & $1.18 \times 10^{4}$ \\
\hline \multirow[t]{2}{*}{$1 P_{1}$} & $\underset{31.22 \%}{\stackrel{\gamma}{\longrightarrow}} 1^{3} S_{1} \underset{100 \%}{\stackrel{\gamma}{\longrightarrow}} B_{c}$ & $5.73 \times 10^{3}$ \\
\hline & $\underset{68.78 \%}{\stackrel{\gamma}{\longrightarrow}} B_{c}$ & $1.26 \times 10^{4}$ \\
\hline $1^{3} P_{0}$ & $\underset{100 \%}{\stackrel{\gamma}{\longrightarrow}} 1^{3} S_{1} \underset{100 \%}{\stackrel{\gamma}{\longrightarrow}} B_{c}$ & $1.23 \times 10^{4}$ \\
\hline
\end{tabular}

TABLE XVIII. Decay chains of $2^{3} P_{2}$ states and expected number of events at the LHC.

\begin{tabular}{|c|c|c|}
\hline $\begin{array}{l}\text { Initial } \\
\text { state }\end{array}$ & Decay chain & $\begin{array}{c}\text { Number of } \\
\text { events }\end{array}$ \\
\hline \multirow[t]{11}{*}{$2^{3} P_{2}$} & $\underset{27.03 \%}{\stackrel{\gamma}{\longrightarrow}} 1^{3} S_{1} \underset{100 \%}{\stackrel{\gamma}{\longrightarrow}} B_{c}$ & $9.08 \times 10^{3}$ \\
\hline & $\underset{59.31 \%}{\stackrel{\gamma}{\longrightarrow}} 2^{3} S_{1} \underset{26.76 \%}{\stackrel{\pi \pi}{\longrightarrow}} 1^{3} S_{1} \underset{100 \%}{\stackrel{\gamma}{\longrightarrow}} B_{c}$ & $5.33 \times 10^{3}$ \\
\hline & $\underset{59.31}{\stackrel{\gamma}{\longrightarrow}} 2^{3} S_{1} \underset{28.91}{\stackrel{\gamma}{\longrightarrow}} 1^{3} P_{2} \underset{100 \%}{\stackrel{\gamma}{\longrightarrow}} 1^{3} S_{1} \underset{100 \%}{\stackrel{\gamma}{\longrightarrow}} B_{c}$ & $5.76 \times 10^{3}$ \\
\hline & $\underset{59.31}{\stackrel{\gamma}{\longrightarrow}} 2^{3} S_{1} \underset{9.59 \%}{\stackrel{\gamma}{\longrightarrow}} 1 P_{1} \underset{68.78 \%}{\stackrel{\gamma}{\longrightarrow}} B_{c}$ & $1.31 \times 10^{3}$ \\
\hline & $\underset{59.31}{\stackrel{\gamma}{\longrightarrow}} 2^{3} S_{1} \underset{15.76 \%}{\stackrel{\gamma}{\longrightarrow}} 1 P_{1}^{\prime} \underset{35.56 \%}{\stackrel{\gamma}{\longrightarrow}} B_{c}$ & $1.12 \times 10^{3}$ \\
\hline & $\underset{59.31}{\stackrel{\gamma}{\longrightarrow}} 2^{3} S_{1} \underset{15.76 \%}{\stackrel{\gamma}{\longrightarrow}} 1 P_{1}^{\prime} \underset{64.44 \%}{\stackrel{\gamma}{\longrightarrow}} 1^{3} S_{1} \underset{100 \%}{\stackrel{\gamma}{\longrightarrow}} B_{c}$ & $2.02 \times 10^{3}$ \\
\hline & $\underset{59.31}{\stackrel{\gamma}{\longrightarrow}} 2^{3} S_{1} \underset{16.23 \%}{\stackrel{\gamma}{\longrightarrow}} 1^{3} P_{0} \underset{100 \%}{\stackrel{\gamma}{\longrightarrow}} 1^{3} S_{1} \underset{100 \%}{\stackrel{\gamma}{\longrightarrow}} B_{c}$ & $3.23 \times 10^{3}$ \\
\hline & $\underset{11.34 \%}{\stackrel{\gamma}{\longrightarrow}} 1^{3} D_{3} \underset{99.91 \%}{\stackrel{\gamma}{\longrightarrow}} 1^{3} P_{2} \underset{100 \%}{\stackrel{\gamma}{\longrightarrow}} 1^{3} S_{1} \underset{100 \%}{\stackrel{\gamma}{\longrightarrow}} B_{c}$ & $3.81 \times 10^{3}$ \\
\hline & $\underset{0.86 \%}{\stackrel{\gamma}{\longrightarrow}} 1 D_{2} \underset{91.8 \%}{\stackrel{\gamma}{\longrightarrow}} 1 P_{1} \underset{68.78 \%}{\stackrel{\gamma}{\longrightarrow}} B_{c}$ & $1.82 \times 10^{2}$ \\
\hline & $\underset{1.29 \%}{\stackrel{\gamma}{\longrightarrow}} 1 D_{2}^{\prime} \underset{84.86 \%}{\stackrel{\gamma}{\longrightarrow}} 1 P_{1}^{\prime} \underset{35.56 \%}{\stackrel{\gamma}{\longrightarrow}} B_{c}$ & $1.31 \times 10^{2}$ \\
\hline & $\underset{1.29 \%}{\stackrel{\gamma}{\longrightarrow}} 1 D_{2}^{\prime} \underset{84.86 \%}{\stackrel{\gamma}{\longrightarrow}} 1 P_{1}^{\prime} \underset{64.44 \%}{\stackrel{\gamma}{\longrightarrow}} 1^{3} S_{1} \underset{100 \%}{\stackrel{\gamma}{\longrightarrow}} B_{c}$ & $2.37 \times 10^{2}$ \\
\hline
\end{tabular}

model that incorporates scalar confinement and one gluon exchange spin-dependent interaction. These eigenfunctions were then used to obtain $E 1$ and $M 1$ radiative transitions. Strong decay amplitudes of excited $B_{c}$ states above the $B D$ threshold have been obtained using the modified ${ }^{3} P_{0}$ pair creation model and fitted SHO wave functions. The hadronic transition rates for $B_{c}$ mesons has been predicted using the Kuang-Yan approach. The total decay widths of excited $B_{c}$ states have been predicted by summing the radiative, hadronic, and strong widths. The branching ratios of different final states are estimated by using the total widths. These branching ratios are then combined with production rates at the LHC to estimate the number of
TABLE XIX. Decay chains of $2 P_{1}^{\prime}$ states and expected number of events at the LHC.

\begin{tabular}{|c|c|c|}
\hline $\begin{array}{l}\text { Initial } \\
\text { state }\end{array}$ & Decay chain & $\begin{array}{c}\text { Number of } \\
\text { events }\end{array}$ \\
\hline \multirow[t]{15}{*}{$2 P_{1}^{\prime}$} & $\underset{10.83 \%}{\stackrel{\gamma}{\longrightarrow}} B_{c}$ & $3.64 \times 10^{3}$ \\
\hline & 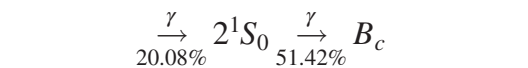 & $3.47 \times 10^{3}$ \\
\hline & 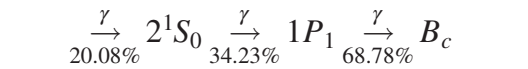 & $1.59 \times 10^{3}$ \\
\hline & $\underset{20.08 \%}{\stackrel{\gamma}{\longrightarrow}} 2^{1} S_{0} \underset{34.23 \%}{\stackrel{\gamma}{\longrightarrow}} 1 P_{1} \underset{31.22}{\stackrel{\gamma}{\longrightarrow}} 1^{3} S_{1} \underset{100 \%}{\stackrel{\gamma}{\longrightarrow}} B_{c}$ & $7.2 \times 10^{2}$ \\
\hline & $\underset{20.08 \%}{\stackrel{\gamma}{\longrightarrow}} 2^{1} S_{0} \underset{14.01}{\stackrel{\gamma}{\longrightarrow}} 1 P_{1}^{\prime} \underset{64.44 \%}{\stackrel{\gamma}{\longrightarrow}} 1^{3} S_{1} \underset{100 \%}{\stackrel{\gamma}{\longrightarrow}} B_{c}$ & $6.09 \times 10^{2}$ \\
\hline & $\underset{37.87 \%}{\stackrel{\gamma}{\longrightarrow}} 2^{3} S_{1} \underset{26.76 \%}{\stackrel{\pi \pi}{\longrightarrow}} 1^{3} S_{1} \underset{100 \%}{\stackrel{\gamma}{\longrightarrow}} B_{c}$ & $3.41 \times 10^{3}$ \\
\hline & $\underset{37.87 \%}{\stackrel{\gamma}{\longrightarrow}} 2^{3} S_{1} \underset{28.91 \%}{\stackrel{\gamma}{\longrightarrow}} 1^{3} P_{2} \underset{100 \%}{\stackrel{\gamma}{\longrightarrow}} 1^{3} S_{1} \underset{100 \%}{\stackrel{\gamma}{\longrightarrow}} B_{c}$ & $3.68 \times 10^{3}$ \\
\hline & $\underset{37.87 \%}{\stackrel{\gamma}{\longrightarrow}} 2^{3} S_{1} \underset{9.59 \%}{\stackrel{\gamma}{\longrightarrow}} 1 P_{1} \underset{68.78 \%}{\stackrel{\gamma}{\longrightarrow}} B_{c}$ & $8.39 \times 10^{2}$ \\
\hline & $\underset{37.87 \%}{\stackrel{\gamma}{\longrightarrow}} 2^{3} S_{1} \underset{15.76 \%}{\stackrel{\gamma}{\rightarrow}} 1 P_{1}^{\prime} \underset{64.44 \%}{\stackrel{\gamma}{\longrightarrow}} 1^{3} S_{1} \underset{100 \%}{\stackrel{\gamma}{\longrightarrow}} B_{c}$ & $1.29 \times 10^{3}$ \\
\hline & $\underset{37.87 \%}{\stackrel{\gamma}{\longrightarrow}} 2^{3} S_{1} \underset{16.23 \%}{\stackrel{\gamma}{\longrightarrow}} 1^{3} P_{0} \underset{100 \%}{\stackrel{\gamma}{\longrightarrow}} 1^{3} S_{1} \underset{100 \%}{\stackrel{\gamma}{\longrightarrow}} B_{c}$ & $2.07 \times 10^{3}$ \\
\hline & 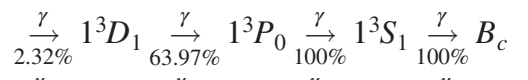 & $4.99 \times 10^{2}$ \\
\hline & 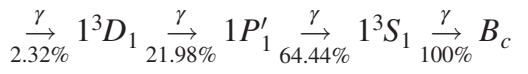 & $1.1 \times 10^{2}$ \\
\hline & $\underset{10.33 \%}{\stackrel{\gamma}{\longrightarrow}} 1 D_{2}^{\prime} \underset{13.32 \%}{\stackrel{\gamma}{\longrightarrow}} 1^{3} P_{2} \underset{100 \%}{\stackrel{\gamma}{\longrightarrow}} 1^{3} S_{1} \underset{100 \%}{\stackrel{\gamma}{\longrightarrow}} B_{c}$ & $4.62 \times 10^{2}$ \\
\hline & $\underset{10.33 \%}{\stackrel{\gamma}{\longrightarrow}} 1 D_{2}^{\prime} \underset{84.86 \%}{\stackrel{\gamma}{\longrightarrow}} 1 P_{1}^{\prime} \underset{35.56 \%}{\stackrel{\gamma}{\longrightarrow}} B_{c}$ & $1.05 \times 10^{3}$ \\
\hline & $\underset{10.33 \%}{\stackrel{\gamma}{\longrightarrow}} 1 D_{2}^{\prime} \underset{84.86 \%}{\stackrel{\gamma}{\longrightarrow}} 1 P_{1}^{\prime} \underset{64.44 \%}{\stackrel{\gamma}{\longrightarrow}} 1^{3} S_{1} \underset{100 \%}{\stackrel{\gamma}{\longrightarrow}} B_{c}$ & $1.9 \times 10^{3}$ \\
\hline
\end{tabular}

TABLE XX. Decay chains of $2 P_{1}$ states and expected number of events at the LHC.

\begin{tabular}{|c|c|c|}
\hline $\begin{array}{l}\text { Initial } \\
\text { state }\end{array}$ & Decay chain & $\begin{array}{c}\text { Number of } \\
\text { events }\end{array}$ \\
\hline \multirow[t]{11}{*}{$2 P_{1}$} & $\underset{23.16 \%}{\stackrel{\gamma}{\longrightarrow}} B_{c}$ & $4.25 \times 10^{3}$ \\
\hline & $\underset{9.97 \%}{\stackrel{\gamma}{\longrightarrow}} 1^{3} S_{1} \underset{100 \%}{\stackrel{\gamma}{\longrightarrow}} B_{c}$ & $1.83 \times 10^{3}$ \\
\hline & $\underset{39.02 \%}{\stackrel{\gamma}{\longrightarrow}} 2^{1} S_{0} \underset{51.42 \%}{\stackrel{\pi \pi}{\longrightarrow}} B_{c}$ & $3.68 \times 10^{3}$ \\
\hline & $\underset{39.02 \%}{\stackrel{\gamma}{\longrightarrow}} 2^{1} S_{0} \underset{34.23 \%}{\stackrel{\gamma}{\longrightarrow}} 1 P_{1} \underset{68.78 \%}{\stackrel{\gamma}{\longrightarrow}} B_{c}$ & $1.69 \times 10^{3}$ \\
\hline & $\underset{39.02 \%}{\stackrel{\gamma}{\longrightarrow}} 2^{1} S_{0} \underset{34.23 \%}{\stackrel{\gamma}{\longrightarrow}} 1 P_{1} \underset{31.22 \%}{\stackrel{\gamma}{\longrightarrow}} 1^{3} S_{1} \underset{100 \%}{\stackrel{\gamma}{\longrightarrow}} B_{c}$ & $7.66 \times 10^{2}$ \\
\hline & $\underset{39.02 \%}{\stackrel{\gamma}{\longrightarrow}} 2^{1} S_{0} \underset{14.01 \%}{\stackrel{\gamma}{\rightarrow}} 1 P_{1}^{\prime} \underset{64.44 \%}{\stackrel{\gamma}{\longrightarrow}} 1^{3} S_{1} \underset{100 \%}{\stackrel{\gamma}{\longrightarrow}} B_{c}$ & $6.47 \times 10^{2}$ \\
\hline & $\underset{18.41 \%}{\stackrel{\gamma}{\longrightarrow}} 2^{3} S_{1} \underset{26.76 \%}{\stackrel{\pi \pi}{\longrightarrow}} 1^{3} S_{1} \underset{100 \%}{\stackrel{\gamma}{\longrightarrow}} B_{c}$ & $9.04 \times 10^{2}$ \\
\hline & $\underset{18.41 \%}{\stackrel{\gamma}{\longrightarrow}} 2^{3} S_{1} \underset{28.91 \%}{\stackrel{\gamma}{\rightarrow}} 1^{3} P_{2} \underset{100 \%}{\stackrel{\gamma}{\longrightarrow}} 1^{3} S_{1} \underset{100 \%}{\stackrel{\gamma}{\longrightarrow}} B_{c}$ & $9.77 \times 10^{2}$ \\
\hline & $9 . \underset{9.27 \%}{\stackrel{\gamma}{\longrightarrow}} 1 D_{2} \underset{8.12 \%}{\stackrel{\gamma}{\longrightarrow}} 1^{3} P_{2} \underset{100 \%}{\stackrel{\gamma}{\longrightarrow}} 1^{3} S_{1} \underset{100 \%}{\stackrel{\gamma}{\longrightarrow}} B_{c}$ & $1.38 \times 10^{2}$ \\
\hline & $9.27 \%$ & $1.07 \times 10^{3}$ \\
\hline & $\underset{9.27 \%}{\stackrel{\gamma}{\longrightarrow}} 1 D_{2} \underset{91.8 \%}{\stackrel{\gamma}{\longrightarrow}} 1 P_{1} \underset{31.22 \%}{\stackrel{\gamma}{\longrightarrow}} 1^{3} S_{1} \underset{100 \%}{\stackrel{\gamma}{\longrightarrow}} B_{c}$ & $4.88 \times 10^{2}$ \\
\hline
\end{tabular}


TABLE XXI. Decay chains of $2^{3} P_{0}$ states and expected number of events at the LHC.

\begin{tabular}{|c|c|c|}
\hline Initial state & Decay chain & Number of events \\
\hline \multirow[t]{8}{*}{$2^{3} P_{0}$} & $\underset{35.87 \%}{\stackrel{\gamma}{\longrightarrow}} 1^{3} S_{1} \underset{100 \%}{\stackrel{\gamma}{\longrightarrow}} B_{c}$ & $5.47 \times 10^{3}$ \\
\hline & $\underset{55.38 \%}{\stackrel{\gamma}{\longrightarrow}} 2^{3} S_{1} \underset{26.76 \%}{\stackrel{\pi \pi}{\longrightarrow}} 1^{3} S_{1} \underset{100 \%}{\stackrel{\gamma}{\longrightarrow}} B_{c}$ & $2.26 \times 10^{3}$ \\
\hline & $\underset{55.38 \%}{\stackrel{\gamma}{\longrightarrow}} 2^{3} S_{1} \underset{28.91 \%}{\stackrel{\gamma}{\longrightarrow}} 1^{3} P_{2} \underset{100 \%}{\stackrel{\gamma}{\longrightarrow}} 1^{3} S_{1} \underset{100 \%}{\stackrel{\gamma}{\longrightarrow}} B_{c}$ & $2.44 \times 10^{3}$ \\
\hline & $\underset{55.38 \%}{\stackrel{\gamma}{\longrightarrow}} 2^{3} S_{1} \underset{15.76}{\stackrel{\gamma}{\rightarrow}} 1 P_{1}^{\prime} \underset{64.44 \%}{\stackrel{\gamma}{\longrightarrow}} 1^{3} S_{1} \underset{100 \%}{\stackrel{\gamma}{\longrightarrow}} B_{c}$ & $8.57 \times 10^{2}$ \\
\hline & $\underset{55.38 \%}{\stackrel{\gamma}{\longrightarrow}} 2^{3} S_{1} \underset{16.23 \%}{\stackrel{\gamma}{\longrightarrow}} 1^{3} P_{0} \underset{100 \%}{\stackrel{\gamma}{\longrightarrow}} 1^{3} S_{1} \underset{100 \%}{\stackrel{\gamma}{\longrightarrow}} B_{c}$ & $1.37 \times 10^{3}$ \\
\hline & $\underset{8.74 \%}{\stackrel{\gamma}{\longrightarrow}} 1^{3} D_{1} \underset{63.97 \%}{\stackrel{\gamma}{\longrightarrow}} 1^{3} P_{0} \underset{100 \%}{\stackrel{\gamma}{\longrightarrow}} 1^{3} S_{1} \underset{100 \%}{\stackrel{\gamma}{\longrightarrow}} B_{c}$ & $8.52 \times 10^{2}$ \\
\hline & $\underset{8.74 \%}{\stackrel{\gamma}{\longrightarrow}} 1^{3} D_{1} \underset{12.1 \%}{\stackrel{\gamma}{\longrightarrow}} 1 P_{1} \underset{68.78 \%}{\stackrel{\gamma}{\longrightarrow}} B_{c}$ & $1.61 \times 10^{2}$ \\
\hline & $\underset{8.74 \%}{\stackrel{\gamma}{\rightarrow}} 1^{3} D_{1} \underset{21.98 \%}{\stackrel{\gamma}{\longrightarrow}} 1 P_{1}^{\prime} \underset{64.44 \%}{\stackrel{\gamma}{\rightarrow}} 1^{3} S_{1} \underset{100 \%}{\stackrel{\gamma}{\longrightarrow}} B_{c}$ & $1.89 \times 10^{2}$ \\
\hline
\end{tabular}

TABLE XXII. Decay chains of $1 D$ states and expected number of events at the LHC.

\begin{tabular}{|c|c|c|}
\hline Initial state & Decay chain & Number of events \\
\hline $1^{3} D_{3}$ & $\underset{99.91 \%}{\stackrel{\gamma}{\longrightarrow}} 1^{3} P_{2} \underset{100 \%}{\stackrel{\gamma}{\longrightarrow}} 1^{3} S_{1} \underset{100 \%}{\stackrel{\gamma}{\longrightarrow}} B_{c}$ & $2.92 \times 10^{3}$ \\
\hline \multirow[t]{3}{*}{$1 D_{2}^{\prime}$} & $\underset{13.32 \%}{\stackrel{\gamma}{\longrightarrow}} 1^{3} P_{2} \underset{100 \%}{\stackrel{\gamma}{\longrightarrow}} 1^{3} S_{1} \underset{100 \%}{\stackrel{\gamma}{\longrightarrow}} B_{c}$ & $3.9 \times 10^{2}$ \\
\hline & $\underset{84.86}{\stackrel{\gamma}{\longrightarrow}} 1 P_{1}^{\prime} \underset{35.56 \%}{\stackrel{\gamma}{\longrightarrow}} B_{c}$ & $8.83 \times 10^{2}$ \\
\hline & $\underset{84.86 \%}{\stackrel{\gamma}{\longrightarrow}} 1 P_{1}^{\prime} \underset{64.44 \%}{\stackrel{\gamma}{\rightarrow}} 1^{3} S_{1} \underset{100 \%}{\stackrel{\gamma}{\rightarrow}} B_{c}$ & $1.6 \times 10^{3}$ \\
\hline \multirow[t]{3}{*}{$1 D_{2}$} & 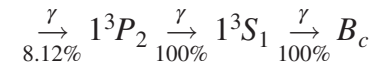 & $2.38 \times 10^{2}$ \\
\hline & $\underset{91.8 \%}{\stackrel{\gamma}{\longrightarrow}} 1 P_{1} \underset{68.78 \%}{\stackrel{\gamma}{\longrightarrow}} B_{c}$ & $1.85 \times 10^{3}$ \\
\hline & $\underset{91.8 \%}{\stackrel{\gamma}{\longrightarrow}} 1 P_{1} \underset{31.22 \%}{\stackrel{\gamma}{\longrightarrow}} 1^{3} S_{1} \underset{100 \%}{\stackrel{\gamma}{\longrightarrow}} B_{c}$ & $8.39 \times 10^{2}$ \\
\hline \multirow[t]{5}{*}{$1^{3} D_{1}$} & $\underset{63.97 \%}{\stackrel{\gamma}{\longrightarrow}} 1^{3} P_{0} \underset{100 \%}{\stackrel{\gamma}{\longrightarrow}} 1^{3} S_{1} \underset{100 \%}{\stackrel{\gamma}{\longrightarrow}} B_{c}$ & $1.87 \times 10^{3}$ \\
\hline & $\underset{12.1 \%}{\stackrel{\gamma}{\longrightarrow}} 1 P_{1} \underset{68.78 \%}{\stackrel{\gamma}{\longrightarrow}} B_{c}$ & $2.45 \times 10^{2}$ \\
\hline & $\underset{12.1 \%}{\stackrel{\gamma}{\longrightarrow}} 1 P_{1} \underset{31.22 \%}{\stackrel{\gamma}{\longrightarrow}} 1^{3} S_{1} \underset{100 \%}{\stackrel{\gamma}{\longrightarrow}} B_{c}$ & $1.11 \times 10^{2}$ \\
\hline & $\underset{21.98 \%}{\stackrel{\gamma}{\longrightarrow}} 1 P_{1}^{\prime} \underset{35.56 \%}{\stackrel{\gamma}{\longrightarrow}} B_{c}$ & $2.29 \times 10^{2}$ \\
\hline & $\underset{21.98 \%}{\stackrel{\gamma}{\longrightarrow}} 1 P_{1}^{\prime} \underset{64.44 \%}{\stackrel{\gamma}{\rightarrow}} 1^{3} S_{1} \underset{100 \%}{\stackrel{\gamma}{\longrightarrow}} B_{c}$ & $4.15 \times 10^{2}$ \\
\hline
\end{tabular}

events of various decay chains of excited $B_{c}$ states. We expect that the predictions presented in this work will help experimentalists find the excited $B_{c}$ states at the LHC and measure their properties.

\section{ACKNOWLEDGMENTS}

F. A. acknowledges the financial support of HEC of Pakistan through Project No. 20-4500/NRPU/R\&D/HEC/14/727. 
[1] F. Abe et al. (CDF Collaboration), Phys. Rev. Lett. 81, 2432 (1998).

[2] R. Aaij et al. (LHCb Collaboration), Phys. Rev. Lett. 108, 251802 (2012).

[3] V. M. Abazov et al. (D0 Collaboration), Phys. Rev. Lett. 101, 012001 (2008).

[4] G. Aad et al. (ATLAS Collaboration), Phys. Rev. Lett. 113, 212004 (2014).

[5] J. Ferretti and E. Santopinto, Phys. Rev. D 97, 114020 (2018).

[6] D. Ebert, R. N. Faustov, and V. O. Galkin, Phys. Rev. D 67, 014027 (2003).

[7] X.-H. Yuan, H.-W. Ke, Y.-B. Ding, and X.-Q. Li, Chin. Phys. C 36, 117 (2012).

[8] S. Godfrey, Phys. Rev. D 70, 054017 (2004).

[9] A. P. Monteiro, M. Bhat, and K. B. Vijaya Kumar, Phys. Rev. D 95, 054016 (2017).

[10] M. Bhat, A. P. Monteiro, and K. B. Vijaya Kumar, Int. J. Mod. Phys. E 26, 1750037 (2017).

[11] A. Abd El-Hady, J. R. Spence, and J. P. Vary, Phys. Rev. D 71, 034006 (2005).

[12] H.-W. Ke and X.-Q. Li, Sci. China Phys. Mech. Astron. 53, 2019 (2010).

[13] T. Barnes, S. Godfrey, and E. S. Swanson, Phys. Rev. D 72, 054026 (2005).

[14] I. Asghar, B. Masud, E. S. Swanson, F. Akram, and M. A. Sultan, Eur. Phys. J. A 54, 127 (2018).

[15] N. Akbar, M. A. Sultan, B. Masud, and F. Akram, Phys. Rev. D 95, 074018 (2017).

[16] M. Tanabashi et al. (Particle Data Group), Phys. Rev. D 98, 030001 (2018).

[17] R. N. Cahn and J. D. Jackson, Phys. Rev. D 68, 037502 (2003).

[18] M. Atif Sultan, N. Akbar, B. Masud, and F. Akram, Phys. Rev. D 90, 054001 (2014).

[19] L. Micu, Nucl. Phys. B10, 521 (1969).

[20] Yu. S. Kalashnikova, Phys. Rev. D 72, 034010 (2005).

[21] J. Ferretti, G. Galata, E. Santopinto, and A. Vassallo, Phys. Rev. C 86, 015204 (2012).

[22] T. Barnes, F. E. Close, P. R. Page, and E. S. Swanson, Phys. Rev. D 55, 4157 (1997).
[23] H. G. Blundell and S. Godfrey, Phys. Rev. D 53, 3700 (1996).

[24] T. Barnes, N. Black, and P. R. Page, Phys. Rev. D 68, 054014 (2003).

[25] J. Ferretti and E. Santopinto, Phys. Rev. D 90, 094022 (2014).

[26] S. Godfrey and K. Moats, Phys. Rev. D 92, 054034 (2015).

[27] F. E. Close and E. S. Swanson, Phys. Rev. D 72, 094004 (2005).

[28] S. Godfrey and K. Moats, Phys. Rev. D 93, 034035 (2016).

[29] Y. Sun, Q.-T. Song, D.-Y. Chen, X. Liu, and S.-L. Zhu, Phys. Rev. D 89, 054026 (2014).

[30] S. Godfrey, K. Moats, and E. S. Swanson, Phys. Rev. D 94, 054025 (2016).

[31] E. S. Ackleh, T. Barnes, and E. S. Swanson, Phys. Rev. D 54, 6811 (1996).

[32] W. Kwong and J. L. Rosner, Phys. Rev. D 38, 279 (1988).

[33] V. A. Novikov, L. B. Okun, M. A. Shifman, A. I. Vainshtein, M. B. Voloshin, and V. I. Zakharov, Phys. Rep. 41, 1 (1978).

[34] T. M. Yan, Phys. Rev. D 22, 1652 (1980).

[35] Y. P. Kuang and T. M. Yan, Phys. Rev. D 24, 2874 (1981).

[36] K. Cheung, Phys. Rev. Lett. 71, 3413 (1993).

[37] K. Cheung and T. C. Yuan, Phys. Lett. B 325, 481 (1994).

[38] K. Cheung and T. C. Yuan, Phys. Rev. D 53, 1232 (1996).

[39] K. Cheung and T. C. Yuan, Phys. Rev. D 53, 3591 (1996).

[40] C. H. Chang and Y. Q. Chen, Phys. Rev. D 48, 4086 (1993).

[41] C. H. Chang and Y. Q. Chen, G. P. Han, and H. T. Jiang, Phys. Lett. B 364, 78 (1995).

[42] C. H. Chang and Y. Q. Chen, G. P. Han, and R. J. Oakes, Phys. Rev. D 54, 4344 (1996).

[43] C. H. Chang, J. X. Wang, and X. G. Wu, Phys. Rev. D 70, 114019 (2004).

[44] K. Kolodziej, A. Leike, and R. Rückl, Phys. Lett. B 355, 337 (1995).

[45] A. V. Berezhnoy, V. V. Kiselev, and A. K. Likhoded, Z. Phys. A 356, 79 (1996); S. P. Baranov, Phys. Rev. D 56, 3046 (1997).

[46] C. H. Chang, H. F. Fu, G. L. Wang, and Z. JinMei, Sci China Phys. Mech. Astron. 58, 1 (2015). 